\title{
MULTI-ZONE MODELING OF THE PULSAR WIND NEBULA HESS J1825-137
}

\author{
ADAM VAN ETTEN ${ }^{1}$ AND ROGER W. ROMANI ${ }^{1}$ \\ Draft version August 19, 2011
}

\begin{abstract}
The pulsar wind nebula associated with PSR J1826-1334, HESS J1825-137, is a bright very high energy source with an angular extent of $\sim 1^{\circ}$ and spatially-resolved spectroscopic TeV measurements. The gammaray spectral index is observed to soften with increasing distance from the pulsar, likely the result of cooling losses as electrons traverse the nebula. We describe analysis of X-ray data of the extended nebula, as well as 3-D time-dependent spectral energy distribution modeling, with emphasis on the spatial variations within HESS J1825-137. The multi-wavelength data places significant constraints on electron injection, transport, and cooling within the nebula. The large size and high nebular energy budget imply a relatively rapid initial pulsar spin period of $13 \pm 7 \mathrm{~ms}$ and an age of $40 \pm 9 \mathrm{kyr}$. The relative fluxes of each VHE zone can be explained by advective particle transport with a radially decreasing velocity profile with $v(r) \propto r^{-0.5}$. The evolution of the cooling break requires an evolving magnetic field which also decreases radially from the pulsar, $B(r, t) \propto r^{-0.7} \dot{E}(t)^{1 / 2}$. Detection of $10 \mathrm{TeV}$ flux $\sim 80 \mathrm{pc}$ from the pulsar requires rapid diffusion of high energy particles with $\tau_{\text {esc }} \approx 90(R / 10 \mathrm{pc})^{2}\left(\mathrm{E}_{\mathrm{e}} / 100 \mathrm{TeV}\right)^{-1}$ year, contrary to the common assumption of toroidal magnetic fields with strong magnetic confinement. The model predicts a rather uniform Fermi LAT surface brightness out to $\sim 1^{\circ}$ from the pulsar, in good agreement with the recently discovered LAT source centered $0.5^{\circ}$ southwest of PSR J1826-1334 with extension $0.6 \pm 0.1^{\circ}$.
\end{abstract}

Subject headings: pulsars: individual (PSR J1826-1334) - X-rays: general

\section{INTRODUCTION}

Pulsar wind nebulae (PWNe) confine magnetized pulsar winds via a termination shock, whereupon particles are reaccelerated to extremely high energies and emit radiation across the electromagnetic spectrum. Many PWNe are spatially resolved in the radio, X-ray, and even very high energy (VHE) wavebands, and thereby provide an excellent laboratory to study not only pulsar winds and dynamics, but also shock processes, the ambient medium, magnetic field evolution, and particle transport.

An archetypal PWN system is HESS J1825-137 associated with the energetic pulsar PSR J1826-1334, which possesses an energy-dependent $\mathrm{TeV}$ morphology thought to be caused by relic electrons from earlier epochs (Aharonian et al. 2006). This system is also a prime example of offset VHE PWNe, with the H.E.S.S. flux centered $0.3^{\circ}$ south of the pulsar and VHE emission extending $1.2^{\circ}$ to the south. The TeV emission softens from a photon index of $\Gamma=1.8$ near the pulsar to $\Gamma=2.5$ in the outer reaches of the nebula.

PSR J1826-1334 was detected in the Jodrell Bank $20 \mathrm{~cm}$ radio survey (Clifton et al. 1992), with period $101 \mathrm{~ms}$, spindown power $\dot{E}=2.8 \times 10^{36} \mathrm{erg} \mathrm{s}^{-1}$, and characteristic age $\tau_{c}=21.4 \mathrm{kyr}$. The dispersion measure distance of the pulsar is $\sim 3.9 \mathrm{kpc}$ (Cordes \& Lazio 2002). X-ray observations of the PWN with XMM-Newton showed a compact core with a hard photon index $\left(\Gamma=1.6_{-0.2}^{+0.1}\right)$ of size $30^{\prime \prime}$ embedded in a larger diffuse structure of extension $\sim 5^{\prime}$ extending to the south of the pulsar with a softer photon index of $\Gamma \approx 2.3$ (Gaensler et al. 2003). Chandra observations by Pavlov et al. (2008) revealed the compact core to be composed of a bright inner component $\approx 7^{\prime \prime} \times 3^{\prime \prime}$ surrounded by an outer component elongated in the east-west direction. More recent X-ray observations with Suzaku revealed diffuse X-ray emission of

\footnotetext{
${ }^{1}$ Department of Physics, Stanford University, Stanford, CA 94305
}

$\Gamma \approx 2.0$ extending $15^{\prime}$ south of the pulsar with little sign of spectral softening (Uchivama et al. 2009). HESS J1825-137 has not been detected in the radio (Osborne et al. 2009).

Using archival VLA observations of PSR J1826-1334 Pavlov et al. (2008) measured a proper motion of $440 d_{4} \mathrm{kms}^{-1}$ (with distance $d=4 d_{4} \mathrm{kpc}$ ) at a position angle $\approx 100 \mathrm{deg}$ east of north. This places the pulsar birthsite some $8^{\prime}=9 d_{4}$ pc to the west for an age of $20 \mathrm{kyr}$ (see Figure 1), and rules out pulsar proper motion as the cause of the large southerly extension of HESS J1825-127. Gaensler et al. (2003) advocated that the offset is instead likely the result of an asymmetric reverse shock returning from the north, as proposed by Blondin et al. (2001) to explain the similar structure observed in Vela-X. Indeed, Lemiere et al. (2006) found a compelling spatial correlation between a molecular cloud discovered in $C O$ studies and the VHE source. The distance to this cloud is measured as $4 \mathrm{kpc}$, and with a total mass of $\approx 4 \times 10^{5} M_{\odot}$ naturally provides the ISM inhomogeneity to the north required for the reverse shock scenario. The fact that emission extends roughly perpendicular to the pulsar motion suggests that the reverse shock velocity must be significantly greater than the pulsar proper motion.

The Fermi Large Area Telescope (LAT) recently detected HESS J1825-137 using 20 months of survey data (Grondin et al. 2011). The gamma-ray emission detected by the LAT boasts a similar size to the H.E.S.S. source, with extension $0.56^{\circ} \pm 0.07^{\circ}$ for an assumed Gaussian model. The $1-100 \mathrm{GeV}$ LAT spectrum of this source is well described by a power-law with a spectral index of $1.4 \pm 0.2$. Single-zone spectral energy distribution (SED) modeling of the X-ray and gamma-ray data showed that $\approx 5 \times 10^{49}$ erg injected in the form of electrons evolved over $25 \mathrm{kyr}$ could reproduce the broadband aggregate spectrum, and that the hard LAT spectrum was consistent with both a simple power-law electron injection spectrum, and with a relativistic Maxwellian with a 
power-law tail (Grondin et al. 2011).

In this paper we describe SED modeling of the X-ray and gamma-ray data, with particular emphasis on the spatial variations in spectra. In Section 2 we overview the X-ray and VHE data, Section 3 details the SED model, Section 4 describes results of SED fitting, and Section 5 discusses the conclusions that can be drawn from model fitting.

\section{DATA ANALYSIS}

\subsection{Suzaku Data}

Diffuse Suzaku X-ray emission surrounding PSR J1826-1334 was analyzed by Uchiyama et al. (2009). The $18^{\prime}$ Suzaku XIS field of view is broad enough to encompass the bright core of HESS J1825-137, and Uchiyama et al. (2009) reported emission nearly to the edge of all four XIS chips. Concurrently, a dedicated background pointing $35^{\prime}$ northeast of the initial exposure and outside the H.E.S.S. emission region was used for spectral backgrounds. More recently, a greater portion of the H.E.S.S. excess was covered by a grid of three Suzaku pointings (unpublished at the time of submission) to the South and West of the initial pointing (see Table 1). A further Suzaku exposure centered on PSR J1826-1334 (also unpublished) expanded the data coverage of the PWN core. These five images therefore cover $\approx 40^{\prime} \times 40^{\prime}$ of the brightest portion of HESS J1825-137, and are listed in Table 1. A number of point sources are observed in the Suzaku mosaic, five of which (A-E) were noted by Uchiyama et al. (2009). These sources are listed in Table 2, as well as displayed in Figure 1. Sources E and $\mathrm{N}$ have no cataloged counterpart.

We utilize the standard pipeline screened events, and analyze the XIS chips with XSelect version 2.4. The four most recent pointings occurred after the loss of the XIS2 chip, so these pointings include the two remaining front side illuminated chips (XIS0, XIS3) as well as the back side illuminated chip (XIS1). All observations operate the XIS in normal clocking full window mode, with the data split between two editing modes: $3 \times 3$ and $5 \times 5$. An exposure corrected mosaic of the five Suzaku exposures is shown in Figure 1. Diffuse

TABLE 1

Suzaku DATA

\begin{tabular}{rccccc}
\hline \hline No. & Obs ID & Date & Pointing $^{\mathrm{a}}$ & Location & Time (ks) \\
\hline 0 & 501045010 & $2006-10-19$ & $276.90,-13.29$ & BG & 52.1 \\
1 & 501044010 & $2006-10-17$ & $276.51,-13.71$ & H.E.S.S. core & 50.3 \\
2 & 503028010 & $2008-10-15$ & $276.50,-14.01$ & S of obs \#1 & 57.2 \\
3 & 503029010 & $2008-10-17$ & $276.20,-13.72$ & W of obs \#1 & 57.2 \\
4 & 503030010 & $2008-10-19$ & $276.19,-13.99$ & SW of obs \#1 & 57.2 \\
5 & 503086010 & $2009-03-19$ & $276.57,-13.59$ & PSR & 52.1 \\
\hline
\end{tabular}

${ }^{\text {a }}$ RA, Dec (degrees)

TABLE 2

$\mathrm{X}$-RAY POINT SOURCES

\begin{tabular}{|c|c|c|c|}
\hline Source & Name/Location $^{\mathrm{a}}$ & Source & Name/Location ${ }^{\mathrm{a}}$ \\
\hline A & 2XMM J182557.9-134755 & $\mathrm{H}$ & 2XMM J182539.9-133130 \\
\hline $\mathrm{B}$ & 2XMM J182617.1-134111 & I & 2RXP J182535.2-135049 \\
\hline $\mathrm{C}$ & 2XMM J182629.5-133648 & $\mathrm{J}$ & 2RXP J182458.3-133747 \\
\hline $\mathrm{D}$ & 2XMM J182620.9-134426 & $\mathrm{K}$ & 2RXP J182509.9-134621 \\
\hline $\mathrm{E}$ & $18: 26: 12-13: 48: 33$ & $\mathrm{~L}$ & 2RXP J182601.4-140423 \\
\hline $\mathrm{F}$ & 2RXP J182640.4-133911 & $\mathrm{M}$ & 2RXP J182436.5-140429 \\
\hline G & 2RXP J182642.6-133625 & $\mathrm{N}$ & $18: 24: 14-13: 58: 15$ \\
\hline
\end{tabular}

a RA, Dec (Sexagesimal) emission clearly extends $\sim 18^{\prime}$ south of the pulsar position, with very little emission beyond this, or to the north of the pulsar.

\subsection{H.E.S.S. Data}

The twelve H.E.S.S. spectral extraction regions extend in $6^{\prime}$ chunks $1.2^{\circ}$ from the pulsar $\left(0.1^{\circ}=7.0 d_{4} \mathrm{pc}\right)$, covering a $90^{\circ}$ sector from approximately $190^{\circ}-280^{\circ}$, measured counterclockwise from North (Aharonian et al. (2006), Figure 4). While these regions capture the majority of the VHE emission, inspection of the H.E.S.S. excess map indicates that a sizable fraction of the emission lies to the southeast of the pulsar and outside of the extraction regions. An accurate value of the ratio of X-ray to gamma-ray flux is important for modeling purposes (see Section 3.1). Therefore, to account for the missing VHE flux, and to allow better comparison with the Suzaku X-ray data (which extends beyond the $90^{\circ}$ sector) and LAT GeV gamma-ray data (which is drawn from a very large extraction region), we extend the quarter annuli an extra $45^{\circ}$ such that they now cover $145^{\circ}-280^{\circ}$. Barring a full reanalysis of the H.E.S.S. data (which is not possible given the propriety nature of H.E.S.S. data) the best we can do is to scale the amplitude of each slice's spectrum by the ratio of total image counts in the original versus extended region. As a check of this method, we compute the image counts ratios between slices. For the original (unscaled) H.E.S.S. regions, the ratio of image counts between any two regions lies within the error bounds of the published H.E.S.S. flux ratios between those two regions. This lends some credence to the assumption that the flux within a region scales directly with the number of counts in the H.E.S.S. excess map.

The scaling factors are listed in Table 3 (along with X-ray scaling factors, described in Section 2.3). These expanded regions capture $81 \%$ of the counts within the $0.8^{\circ}$ radius region used to extract the aggregate H.E.S.S. spectrum. The VHE flux data points of the SED plots (Figures 6, 7, 9, 11) are therefore taken to be the rescaled points from Figure 4 of Aharonian et al. (2006), and summing the rescaled flux data points of these regions yields data points $\approx 25 \%$ below the aggregate H.E.S.S. data. This is quite close to the expected $19 \%$ difference expected from the counts ratio in the H.E.S.S. excess map. Some of the missing flux is the northeast extension of the nebula, but some is likely contributed by adjacent regions to the north. Aharonian et al. (2006) estimate the systematic error on the flux scale to be $20 \%$, though for SED fitting we utilize statistical errors only. Figure 1 shows the H.E.S.S. excess map and spectral extraction regions.

\subsection{X-ray Spectrum}

Motivated by SED modeling, we extract Suzaku Xray spectra from $6^{\prime}$ wedges to mimic the regions of Aharonian et al. (2006). Spectral fitting is accomplished with Xspec version 12.6. For background subtraction we follow the same procedure as Uchiyama et al. (2009) (a more indepth discussion can be found in this paper) and define the same region as these authors: a $9^{\prime}$ circle on the background exposure, excising $1.8^{\prime}$ radius circles around the four point sources noted by these authors. The non-uniformity of the background is taken into account internally within Xspec. Spectra are extracted with XSelect, while ARF and RMF response files are generated with the xisrmfgen and xissimarfgen functions, which internally take into account the different responses of each chip. The ASCA FTOOL addascaspec 

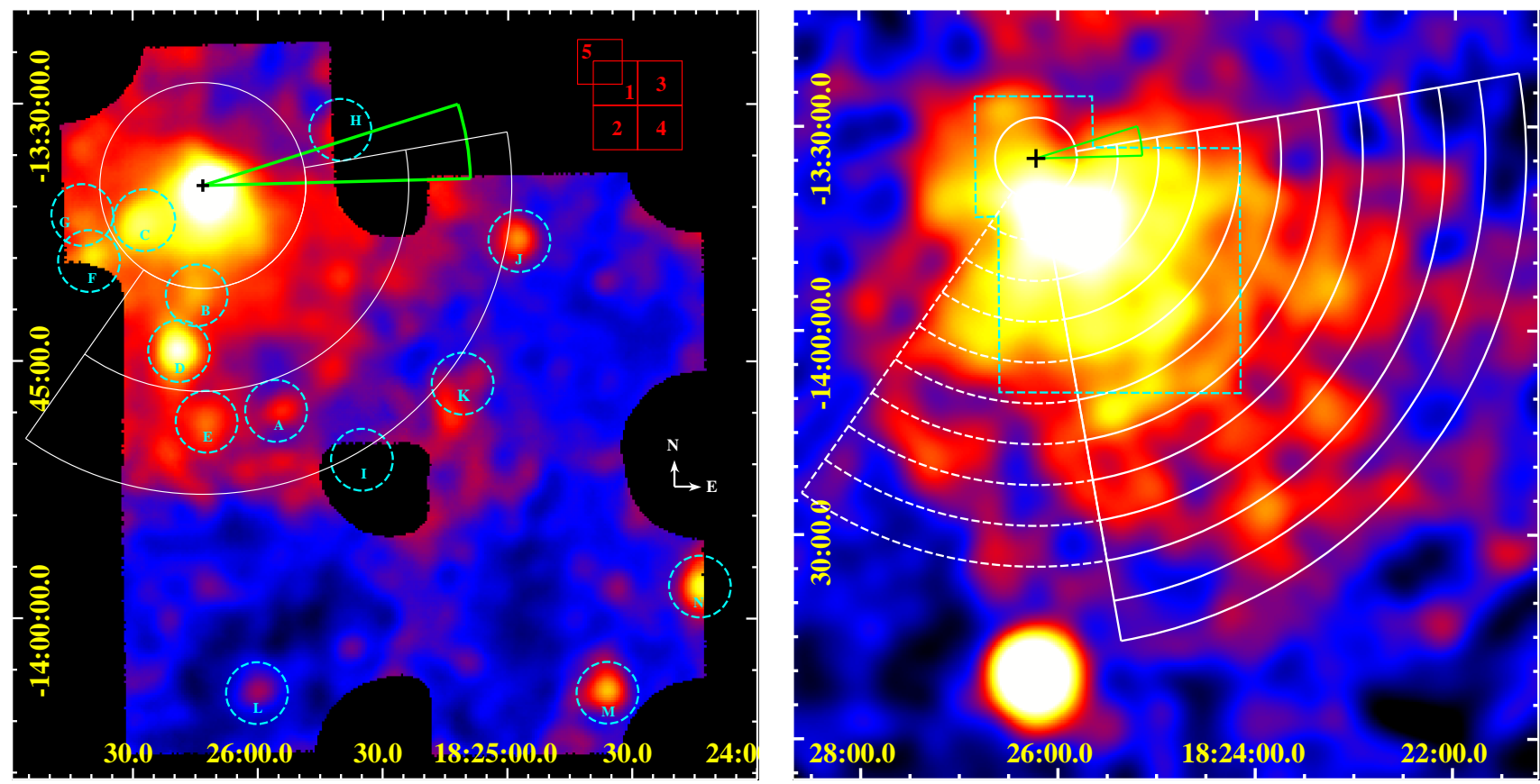

FIG. 1.- Left: Suzaku exposure corrected $1-8 \mathrm{keV}$ counts mosaic of the front side illuminated chips for all five pointings, smoothed with a $1.2^{\prime}$ Gaussian. Point sources (see Table 2) are denoted by $1.8^{\prime}$ radius dashed circles, and the three X-ray spectral extraction regions are shown in solid white. PSR J1826-1334 is indicated by the black cross, and the green wedge indicates the range of possible pulsar birthsites (Pavlov et al. 2008). This green wedge extends some 16', corresponding to the presumed birthsite for an age of $40 \mathrm{kyr}$. The location of each of the five exposures is indicated by the map in the upper right. Right: H.E.S.S. excess counts map showing the VHE spectral extraction regions (solid white), and larger extrapolated regions (dashed white). The Suzaku field of view is shown in cyan. The bright point source to the south is the $\gamma$-ray binary LS 5039.

is utilized to combine the spectra and responses of the XIS front side illuminated chips. The XIS1 back side illuminated chip possesses a markedly different response function from the front side illuminated chips, and so is analyzed in parallel rather than added to the other chips.

Initially, we extract and fit the spectrum of the $1.5^{\prime}$ circle surrounding PSR J1826-1334 titled Region A by Uchiyama et al. (2009). Fitting an absorbed power-law model we achieve a fit well within errors of the parameters reported by these authors $(\Gamma=1.7 \pm 0.1$, unabsorbed $1-8 \mathrm{keV}$ flux of $\left.1.2 \pm 0.2 \times 10^{-12} \mathrm{erg} \mathrm{cm}^{-2} \mathrm{~s}^{-1}\right)$. We subsequently define regions mimicking the inner $6^{\prime}$ circle surrounded by $6^{\prime}$ thick partial annuli shown in Figure 1. From these regions we excise $1.8^{\prime}$ radius circles around the 5 point sources defined by Uchiyama et al. (2009). For the innermost $6^{\prime}$ circle we use both the original dataset (501044010), as well as the most recent dataset (503086010) and jointly fit the absorption

TABLE 3

H.E.S.S. SPECTRAL DATA

\begin{tabular}{ccccc}
\hline \hline Region & X-Ray Scaling & H.E.S.S. Scaling & $\Gamma^{\mathrm{a}}$ & Scaled Flux $^{\mathrm{b}}$ \\
\hline $0^{\prime}-6^{\prime}$ & 1.13 & 1.0 & $1.83 \pm 0.09$ & $2.9 \pm 0.3$ \\
$6^{\prime}-12^{\prime}$ & 1.16 & 1.5 & $1.96 \pm 0.08$ & $4.4 \pm 0.4$ \\
$12^{\prime}-18^{\prime}$ & 1.27 & 1.4 & $2.20 \pm 0.06$ & $6.0 \pm 0.4$ \\
$18^{\prime}-24^{\prime}$ & & 1.4 & $2.25 \pm 0.06$ & $7.4 \pm 0.4$ \\
$24^{\prime}-30^{\prime}$ & 1.4 & $2.32 \pm 0.07$ & $8.7 \pm 0.5$ \\
$30^{\prime}-36^{\prime}$ & 1.4 & $2.37 \pm 0.06$ & $9.7 \pm 0.5$ \\
$36^{\prime}-42^{\prime}$ & 1.3 & $2.36 \pm 0.08$ & $7.4 \pm 0.5$ \\
$42^{\prime}-48^{\prime}$ & 1.4 & $2.41 \pm 0.09$ & $7.6 \pm 0.6$ \\
$48^{\prime}-54^{\prime}$ & & 1.2 & $2.42 \pm 0.09$ & $6.1 \pm 0.5$ \\
$54^{\prime}-60^{\prime}$ & 1.2 & $2.59 \pm 0.13$ & $6.2 \pm 0.6$ \\
$60^{\prime}-66^{\prime}$ & 1.0 & $2.43 \pm 0.09$ & $5.9 \pm 0.5$ \\
$66^{\prime}-72^{\prime}$ & & 1.0 & $2.45 \pm 0.35$ & $2.9 \pm 0.7$ \\
\hline
\end{tabular}

${ }^{\text {a }}$ Data taken from Aharonian et al. (2006), Table 2

b $0.25-10 \mathrm{TeV}$ fluxes in units of $10^{-12} \mathrm{erg} \mathrm{cm}^{-2} \mathrm{~s}^{-1}$ and power-law index. Since the original dataset does not encompass the entirety of the $6^{\prime}$ circle we allow the power-law normalization to vary between the two datasets, though we quote the flux from the entire circle. The $6^{\prime}-12^{\prime}$ region follows the same procedure, using both datasets 501044010 and 503086010. For overlapping regions (exposure 1 and exposure 5) the joint spectral fits to both exposures are completely consistent (albeit with smaller errors) in both index and total flux (when taking into account the differing areas of the regions) with fits to the individual exposures.

All fits are to an absorbed power-law from $1-8 \mathrm{keV}$ with quoted single parameter $68 \%(1 \sigma)$ errors. We fit the $6^{\prime}-12^{\prime}$ region with $N_{H}$ fixed at the value determined in the innermost region, and find values completely consistent (albeit with smaller errors) with fitting with $N_{H}$ free. Though $N_{H}$ may vary over the nebula, we see no evidence of this, and IR maps show no appreciable gradient over the nebula, so for simplicity we adopt a uniform column density. The pulsar will contribute flux to the innermost region, though with a $1-8$ $\mathrm{keV}$ flux of $\sim 2 \times 10^{-14} \mathrm{erg} \mathrm{cm}^{-2} \mathrm{~s}^{-1}$ (Pavlov et al. 2008), the pulsar presents only a minor perturbation to the PWN flux.

The $12^{\prime}-18^{\prime}$ wedge stretches across three different pointings and suffers from a much lower surface brightness than the inner two regions, greatly complicating flux extraction from this region. Due to the limited statistics, we fix the absorption to the best fit value from the innermost region. Extracting flux from the portion of this region which lies within the original pointing (501044010) yields $\mathrm{a} \sim 5 \sigma$ detection. No significant emission further south and west of the pulsar is seen in any of the three exposures. In fact, we can use the apparent lack of flux in the southwest exposure furthest from the pulsar (503030010) to estimate the uncertainty in the background. Selecting a background region from this chip alters flux estimates by $\approx 1 \%$ for Region $1\left(0^{\prime}-6^{\prime}\right), \approx 3 \%$ for Region 2 , 
TABLE 4

X-RAY SPECTRAL FITS

\begin{tabular}{lcccc}
\hline \hline Region & $N_{H^{\mathrm{a}}}$ & $\Gamma$ & Unabs. Flux $^{\mathrm{b}}$ & $\chi^{2} /$ d.o.f. \\
\hline $0^{\prime}-6^{\prime}$ & $1.26 \pm 0.06$ & $1.93 \pm 0.05$ & $4.90_{-0.32}^{+0.34}$ & $196 / 249$ \\
\hline $6^{\prime}-12^{\prime}$ & $1.12_{-0.10}^{+0.11}$ & $2.03 \pm 0.09$ & $3.05_{-0.34}^{+0.39}$ & $144 / 173$ \\
$6^{\prime}-12^{\prime}$ & $1.3^{\mathrm{c}}$ & $2.17 \pm 0.05$ & $3.18 \pm 0.15$ & $146 / 174$ \\
\hline $12^{\prime}-18^{\prime}$ & $1.3^{\mathrm{c}}$ & $1.78 \pm 0.18$ & $1.04_{-0.19}^{+0.22}$ & $44 / 59$ \\
\hline a interstellar absorption $\times 10^{22} \mathrm{~cm}^{-2}$ & & \\
b $1-8 \mathrm{keV}$ fluxes in units of $10^{-12} \mathrm{erg} \mathrm{cm}^{-2} \mathrm{~s}^{-1}$ & \\
c held fixed
\end{tabular}

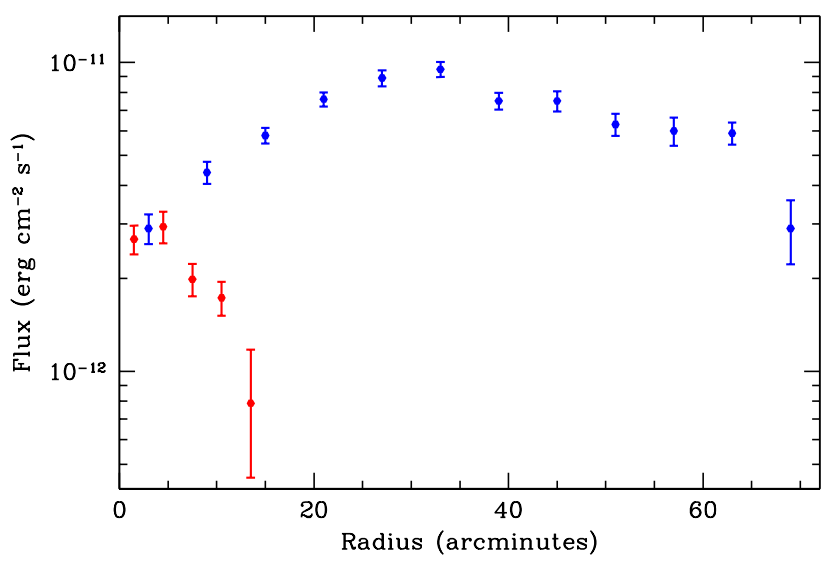

FIG. 2.- Radial flux profile with $1 \sigma$ statistical error bars. Suzaku $1-8$ $\mathrm{keV} \mathrm{X}$-ray flux is shown in red, while the far greater extent of the H.E.S.S. $0.25-10 \mathrm{TeV}$ flux (from Table 3 ) is in blue.

and $\approx 26 \%$ for Region 3 . An additional source of error stems from the uncertainty in the ARF calculation for complex extended sources, which leads to uncertainty in the source flux (Ishisaki et al. 2007), which we estimate at $\approx 10 \%$. Note that background subtraction errors dominate for Zone 3; in fact, the relatively hard spectrum fit here may reflect residual background contamination. In the SED plots we show regrouped $\mathrm{X}$-ray data with the ARF and background systematic errors summed in quadrature with $1 \sigma$ statistical errors.

To be perfectly consistent, for modeling purposes we should utilize identical regions for both X-ray and VHE gamma-ray data. The excised point sources and slight portion of the H.E.S.S. spectral extraction regions which extends beyond the $S u z a k u$ exposures therefore require a small adjustment to the X-ray flux level. The raw X-ray data is therefore rescaled by the fraction of missing area, with the scaling factors shown in Table 3. Table 4 and the SED plots therefore display the rescaled fluxes (with errors adjusted accordingly) in order to remain fully consistent with the H.E.S.S. data.

The X-ray flux falls off rapidly with radial distance from the pulsar, likely the result of both lower magnetic fields as well as cooling losses, as discussed below. Figure 2 shows a radial profile of fluxes in the X-ray and TeV energy bands, clearly indicating the much greater extent of the nebula in the H.E.S.S. regime. For this figure, we fix $N_{H}$ at $1.3 \times 10^{22} \mathrm{~cm}^{-2}$ and extract X-ray spectra from $3^{\prime}$ width regions for greater detail than the $6^{\prime}$ regions shown in Table 4.

\section{SED MODELING}

\subsection{Motivation}

Grondin et al. (2011) applied a one-zone SED model to the new Fermi LAT data of HESS J1825-137 and found an adequate fit to the aggregate gamma-ray and X-ray data. Onezone models can help constrain certain global nebula properties, such as the total energy injected in the form of electrons, the electron spectrum in the uncooled regime, and the mean magnetic field. Yet such models by definition assume a completely homogeneous nebula, contrary to the differing $\mathrm{X}$ ray and VHE sizes and spectral steepening observed in both $\mathrm{X}$-rays and gamma-rays. To further investigate these phenomena we implement a spatially resolved model of HESS J1825-137.

We begin by investigating how simple scaling relations inform model implementation. Starting assumptions include: the source of all particles and magnetic field in the nebula is PSR J1826-1334, ambient photon fields are static, and the nebula is $4 \mathrm{kpc}$ distant. Let us first consider the electrons present in the innermost zone (Zone 1) adjacent the pulsar. In a transverse magnetic field these particles synchrotron radiate photons at mean energy:

$$
E_{\gamma} \approx 2.2\left(E_{e} / 100 \mathrm{TeV}\right)^{2}(B / 10 \mu \mathrm{G}) \mathrm{keV} .
$$

We scale with a low field of $10 \mu \mathrm{G}$ since, as we shall see later, even in the innermost zone the mean field is only $\sim 10 \mu \mathrm{G}$ for the best fit model. Such particles cool rapidly, with a lifetime $(\tau \equiv E / \dot{E})$ of:

$$
\tau_{\text {sync }} \approx 820\left(E_{e} / 100 \mathrm{TeV}\right)^{-1}(B / 10 \mu \mathrm{G})^{-2} \text { year. }
$$

Therefore the cooling timescale for electrons radiating synchrotron photons at mean energy $E_{\gamma}$ is:

$$
\tau_{\text {sync }} \approx 1200\left(E_{\gamma} / 1 \mathrm{keV}\right)^{-1 / 2}(B / 10 \mu \mathrm{G})^{-3 / 2} \text { year. }
$$

The flat $(\Gamma \approx 2) \mathrm{X}$-ray spectrum extending to $10 \mathrm{keV}$ of Region 1 indicates that the electrons responsible for this emission are largely uncooled, implying $\tau \lesssim \tau_{\text {sync }} \approx$ $380(B / 10 \mu \mathrm{G})^{-3 / 2}$ year. The inner region therefore must be dominated by particles recently injected by the pulsar. In the uncooled regime the synchrotron spectral flux scales with the magnetic field and number of electrons as: $F(E)_{\text {sync }} \propto$ $n_{e} \times B^{3 / 2}$. Over timescales short compared to the $21 \mathrm{kyr}$ spindown age of the pulsar the number of electrons $n_{e}$ in Zone 1 is roughly proportional to the time period over which electrons have been injected $\delta t$, so $F(E)_{\text {sync }} \propto \delta t \times B^{3 / 2}$, a relation we will return to momentarily.

Electrons inverse Compton (IC) upscatter CMB photons to a mean energy of:

$$
E_{\gamma} \approx 0.32\left(E_{e} / 10 \mathrm{TeV}\right)^{2} \mathrm{TeV}
$$

For young electrons IC cooling is of little consequence, since $10 \mathrm{TeV}$ electrons scattering off the CMB cool in $\tau_{I C} \approx$ $140\left(E_{e} / 10 \mathrm{TeV}\right)^{-1} \mathrm{kyr}$. We scale to $10 \mathrm{TeV}$ rather than 100 $\mathrm{TeV}$ since at $100 \mathrm{TeV}$ the Thomson regime is no longer realized and Klein-Nishina corrections must be incorporated. For example, while the above scaling relation for inverse Compton cooling holds for electrons with $E_{e} \leq 10 \mathrm{TeV}, 100 \mathrm{TeV}$ electrons cool slowly over $36 \mathrm{kyr}$, rather than the $14 \mathrm{kyr}$ predicted by this relation. We therefore consider only synchrotron cooling in the following discussion. The lowest energy H.E.S.S. point at $E_{\gamma} \approx 0.3 \mathrm{TeV}$ requires electrons of 
energy $E_{e} \approx 10 \mathrm{TeV}$, which synchrotron cool slowly at a rate of $\tau_{\text {sync }} \approx 8200(B / 10 \mu \mathrm{G})^{-2}$ year. Therefore, electrons responsible for the low energy H.E.S.S. points cool quite slowly compared to those responsible for the X-ray flux.

The electrons responsible for the lowest energy H.E.S.S. points (and therefore all the Fermi LAT flux points as well) are radiatively uncooled over time spans of a few thousand years, and provide a good indication of just how many electrons are present in the inner nebula. This is due to the fact that for $E_{e} \leq 10 \mathrm{TeV}$ the Thomson limit holds and IC flux scales linearly with the number of electrons $\left(n_{e}\right)$ present: $F(E)_{I C} \propto n_{e}$. The exact number of electrons at $10 \mathrm{TeV}$ will depend on the electron injection spectrum parameters as well as the injection time $\delta t$, and very weakly on the magnetic field due to feeble synchrotron cooling, so $F(E)_{I C} \propto \delta t$. The X-ray data breaks the degeneracy in magnetic field since $F(E)_{\text {sync }} \propto \delta t \times B^{3 / 2}$, so between X-ray and gamma-ray data the electron injection spectrum, age, and magnetic field are very well constrained. For modeling the data, this means that once an injection spectrum is selected, the gamma-ray flux determines the injection time, while the ratio of X-ray to gamma-ray flux determines the magnetic field.

\subsection{Pulsar Spin-down}

As pulsars spin down, they dissipate rotational kinetic energy according to:

$$
\dot{E}=I \Omega \dot{\Omega},
$$

with $\Omega$ the angular frequency and $I$ the neutron star's moment of inertia, assumed to be $10^{45} \mathrm{~g} \mathrm{~cm}^{2}$. This energy goes into a magnetized particle wind such that for a braking index of $n$ :

$$
\dot{\Omega} \propto \Omega^{n} .
$$

For spin-down via magnetic dipole radiation $n=3$, though $n$ has only been confidently measured for five pulsars, in each case falling between $2<n<3$ (Livingstone et al. (2007) and references therein). Integrating Equation 6 yields the age of the system (Manchester \& Taylor 1977):

$$
T=\frac{P}{(n-1)|\dot{P}|}\left(1-\left(\frac{P_{0}}{P}\right)^{(n-1)}\right),
$$

where $P_{0}$ is the initial spin period, and $\dot{P}$ the period derivative. For $P_{0} \ll P$ and $n=3$ this equation reduces to the characteristic age of the pulsar $\tau_{c} \equiv P / 2 \dot{P}$. The spin-down luminosity of the pulsar evolves as (Pacini \& Salvati 1973):

$$
\dot{E}(t)=\dot{E}_{0}\left(1+\frac{t}{\tau_{0}}\right)^{-\frac{(n+1)}{(n-1)}},
$$

with the initial spin-down timescale defined as:

$$
\tau_{0} \equiv \frac{P_{0}}{(n-1)\left|\dot{P}_{0}\right|} .
$$

Given that the current $P, \dot{P}$, and $\dot{E}$ are known, once an initial period $P_{0}$ and braking index $n$ are selected the age $T$ and spin-down history of the system are determined according to the equations above.

The energy flux in the magnetized wind $\dot{E}$ is split between electromagnetic $\dot{E}_{B}$ and particle energy $\dot{E}_{e}$, with:

$$
\dot{E}_{e}=\eta \dot{E}
$$

$$
\dot{E}_{B}=(1-\eta) \dot{E} .
$$

Their ratio is commonly referred to as the wind magnetization parameter:

$$
\sigma \equiv \dot{E}_{B} / \dot{E}_{e}=(1-\eta) / \eta
$$

with $\sigma$ typically thought to be $\ll 1$ past the termination shock. The particle energy is presumed to go into an electron/positron plasma with an exponentially cutoff power-law spectrum:

$$
\frac{d N}{d E} \propto E^{-p} e^{-E / E_{c u t}} \quad E>E_{m i n},
$$

with $E_{\min }$ the minimum particle energy. We initially select $E_{\text {min }}$ as $100 \mathrm{GeV}$, which is well within the realm of minimum particle energies considered by Kennel \& Coroniti (1984b) in magnetohydrodynamical modeling of the Crab Nebula. The normalization of this power-law varies with time following Equation 8, though we treat the index, minimum energy, and cutoff energy as static. We begin by modeling pure power-law injection, although we later consider an injection spectrum composed of a relativistic Maxwellian with a non-thermal tail, as proposed by Spitkovsky (2008).

\subsection{Spatial Evolution}

Pulsar winds flow out at relativistic speeds until the ram pressure of the wind is balanced by the internal pressure of the PWN at the termination shock. High-resolution X-ray images can help illuminate the location of this termination shock, and Chandra images of the pulsar reveal that the brightest inner PWN component stretches some $7^{\prime \prime} \times 3^{\prime \prime}\left(0.14 \times 0.06 \mathrm{pc}^{2}\right.$ at 4 kpc) (Pavlov et al. 2008), with the short axis roughly oriented toward the pulsar birthsite. The overall extent of the equatorial flow is generally $2-3$ times larger than the termination shock radius ( $\mathrm{Ng} \&$ Romani 2004), and we accordingly adopt a value of $r_{t s}=0.03 \mathrm{pc}$ for the radius of the termination shock.

Past the termination shock the pulsar wind expands radially at a much reduced velocity. In the model of of Kennel \& Coroniti (1984a) the rapid rotation of the pulsar wraps up magnetic field lines, resulting in a primarily toroidal field at and beyond the termination shock. The field direction is therefore approximately perpendicular to the wind flow velocity. Since particles effectively cannot cross toroidal magnetic field lines, the bulk flow of particles predicts an ordered layering of particle ages with increasing distance from the pulsar, of age:

$$
t(r)=\int_{0}^{r} v\left(r^{\prime}\right)^{-1} d r^{\prime}
$$

with $v(r)$ the velocity profile.

On larger scales, we consider the evolution of the parent supernova remnant (SNR) of PSR J1826-1334. Early in the pulsar lifetime the SNR expands freely into the ambient medium, typically at a velocity of $\sim 10,000 \mathrm{~km} \mathrm{~s}^{-1}$. The large $\sim 80 \mathrm{pc}$ size of the VHE PWN and lack of any observed SNR shell led de Jager \& Diannati-Atail (2008) to propose a very large SNR of radius $\approx 120$ pc resulting from an energetic supernova explosion $\left(E_{S N}=3 \times 10^{51} \mathrm{erg}\right)$ of age $\approx 40 \mathrm{kyr}$, and a very low ambient medium density of $n_{0} \approx 0.001 \mathrm{~cm}^{-3}$. Early on, a spherically symmetric PWN expands rapidly into unshocked supernova ejecta with nearly 
constant velocity (van der Swaluw et al. 2001):

$$
\begin{aligned}
v_{p w n} \approx & 3100\left(\frac{\dot{E}_{0}}{10^{39} \mathrm{erg} \mathrm{s}^{-1}}\right)^{1 / 5}\left(\frac{E_{S N}}{3 \times 10^{51} \mathrm{erg}}\right)^{3 / 10} \\
& \times\left(\frac{M_{e j}}{8 M_{\odot}}\right)^{-1 / 2}\left(\frac{t}{1 \mathrm{kyr}}\right)^{1 / 5} \mathrm{~km} \mathrm{~s}^{-1}
\end{aligned}
$$

As the SNR expands and sweeps up more mass, it eventually reaches a point where the swept up ISM material is approximately equal in mass to the mass of the supernova ejecta. At this point the SNR enters the Sedov-Taylor phase as the swept-up material begins to dominate the SNR dynamics. Adopting a uniform density ISM, for the standard corecollapse supernovae assumption of a constant density inner core surrounded by a $\rho \propto r^{-9}$ outer envelope the transition to the Sedov-Taylor phase occurs at (Truelove \& McKee 1999):

$$
\begin{aligned}
t_{S-T} \approx & 7100\left(\frac{E_{S N}}{3 \times 10^{51} \mathrm{erg}}\right)^{-1 / 2}\left(\frac{M_{e j}}{8 M_{\odot}}\right)^{5 / 6} \\
& \times\left(\frac{n_{0}}{0.001 \mathrm{~cm}^{-3}}\right)^{-1 / 3} \text { year. }
\end{aligned}
$$

In this phase a reverse shock is driven deep into the SNR interior, and for a SNR expanding into a homogenous medium the reverse shock will impact the PWN, crushing it and leading to a series of reverberations which can last many thousands of years, as demonstrated compellingly in Gelfand et al. (2009). Calculations by van der Swaluw et al. (2004) yielded an upper limit on the reverse shock collision with the PWN at $t_{r s}<5 t_{S-T}$. A supernova explosion into an evacuated cavity of $n_{0}=0.001 \mathrm{~cm}^{-3}$ therefore yields a maximum PWNreverse shock collision time of $\approx 35 \mathrm{kyr}$ (nearly double the characteristic age of the pulsar), implying that the PWN could to this day be rapidly expanding to the southwest into unshocked SNR ejecta. Of course, reflection off of the molecular cloud discovered by Lemiere et al. (2006) could result in a reverse shock collision on a timescale reduced by an order of magnitude from this estimate.

Axisymmetric 2-D simulations by Blondin et al. (2001) showed that SNR expansion into an inhomogeneous medium leads to a PWN offset from the pulsar position and mixing of the thermal gas of the supernova ejecta and the relativistic gas of the PWN. Rayleigh-Taylor instabilities aid in the mixing and disruption of the nebula as bubbles of swept up material penetrate the PWN. The simulations of Blondin et al. (2001) showed a cometary nebula $\sim 20 \mathrm{kyr}$ after the reverse shock interaction, and nearly complete disruption of the nebula after $\sim 50 \mathrm{kyr}$. These simulations do not take into account the magnetic field that is mixed in with the relativistic particles, and which can dampen instabilities along magnetic field lines. Indeed, recent simulations by Bucciantini et al. (2004) suggest that the growth of instabilities is highly suppressed for high $\sigma$ flows, and significant structure may exist even some $\sim 20$ kyr after reverse shock interaction. This suggests that the ordered layering of particle ages predicted by Equation 13 may remain valid in the case of an anisotropic reverse shock.

If one invokes PWN expansion within a freely expanding SNR to account for the $\sim 80 \mathrm{pc}$ size of the PWN, a mean velocity of $3100 \mathrm{~km} \mathrm{~s}^{-1}$ over $25 \mathrm{kyr}$ is required. While this velocity is quite high for a PWN, it is permitted by Equation 14 for a very low density ambient medium. The $17^{\prime}\left(20 d_{4} \mathrm{pc}\right)$ displacement of the H.E.S.S. centroid south of PSR J1826-1334 (Aharonian et al. 2006) cannot be explained by pure expansion, however, and requires some mechanism to achieve this offset.

One possibility for the southerly displacement is the existence of a powerful jet rapidly transporting particles far from the pulsar. X-ray images show no indication of any such feature, however. In addition, PWN jets are typically aligned with the pulsar spin axis, and a southern jet would be roughly perpendicular to the inferred pulsar proper motion, also typically aligned with the pulsar spin axis.

A more palatable alternative is that the southerly offset from the pulsar is due to crushing by the reverse shock. In this picture the reverse shock quickly reflects off of the molecular cloud to the north, and then impacts the PWN in a few thousand years. The mechanism responsible for the PWN displacement, be it direct interaction with the reverse shock or a resultant anisotropy from the shock, is evidently still at work since the young X-ray emitting electrons are clearly concentrated south of the pulsar. For a reverse shock returning from the North, particles initially north of the pulsar will be displaced south and mixed throughout the nebula. The fact that the H.E.S.S. spectral index of HESS J1825-137 increases monotonically from the pulsar hints that such mixing is likely far from complete. Precisely how the reverse shock crushing and displacement alters any ordered layering of the PWN requires detailed 2-D or 3-D magnetohydrodynamical simulations and is beyond the scope of this paper. We ignore such effects, and hence tacitly assume that for HESS J1825-137 reverse shock interactions serve primarily to displace rather than compress and mix the nebula.

\subsection{Morphology}

The shape of the nebula as viewed from earth resembles a circular sector. While the 3-dimensional morphology of HESS J1825-137 is uncertain, if the morphology results from a shock returning from the north, this implies nebular axial symmetry about the normal to the shock front. One simple geometry which matches the Suzaku and H.E.S.S. morphology is a spherical wedge of opening angle $135^{\circ}$ (solid angle 3.88 steradians) with symmetry axis along the H.E.S.S. symmetry axis $17^{\circ}$ counterclockwise from North. The molecular cloud which presumably gives rise to the assymetric reverse shock is located at the same $4 \mathrm{kpc}$ distance as the pulsar, so we assume that the shock normal is perpendicular to the line of sight. For modeling purposes we split this wedge into twelve 3-D zones which mimic the spectral extraction regions. Given the axisymmetry, the twelve spatial zones form a series of nested bowls, or partial spherical wedges, of thickness $7.0 d_{4}$ pc. Similar to the spectral extraction regions, we label Zone 1 adjacent to the pulsar (extending $0-7 \mathrm{pc}$ ), and Zone 12 the outermost spherical wedge $(77-84 \mathrm{pc}$ from the pulsar). Henceforth we refer to Regions as the 2-D projections on the sky of the 3-D spatial Zones. Figure 3 demonstrates the adopted morphology, with the symmetry axis oriented vertically for clarity. The final volume of each bubble is determined by the spherical wedge morphology discussed above. These volumes are listed in Table 5 .

When projected into the sky, the counts from each bowl will overlap into multiple spectral extraction regions. In order to determining the degree of overlap we perform a Monte Carlo integration by projecting the 3 -D spatial zones onto the plane of the sky. For example, consider Zone 8 extending $49 \mathrm{pc}-56 \mathrm{pc}$ from the pulsar. We populate this zone with a large number of random points, then project each point onto 


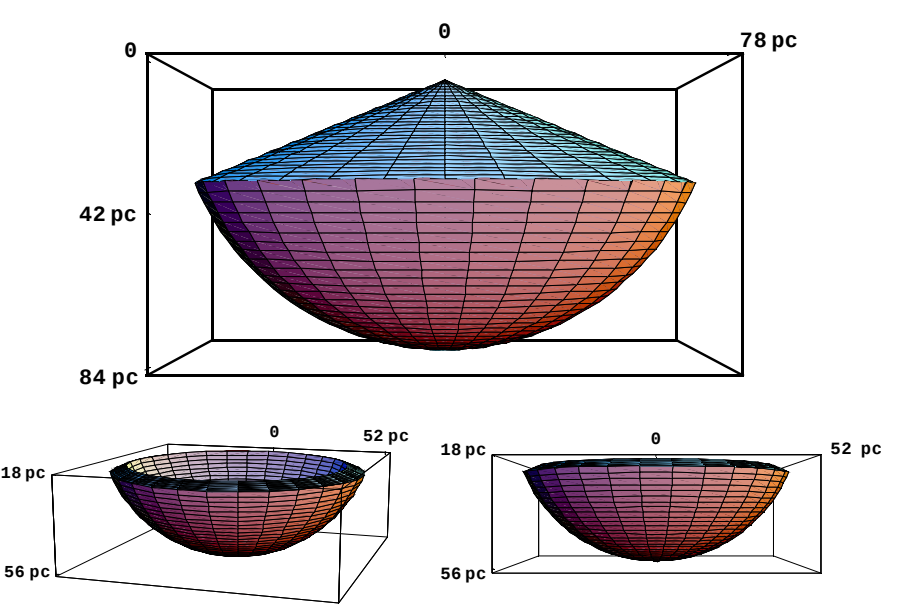

FIG. 3.- Top: Spatial model of HESS J1825-137 as seen from Earth, with the symmetry axis oriented vertically. The pulsar is located at the origin, and the axes indicate the extent of nebula in parsecs. Bottom Left: One of the twelve spatial zones (Zone 8: 49 - 56 pc from the pulsar) plotted with a slightly elevated viewpoint to demonstrate the concave nature of the zone. Bottom Right: The same spatial zone seen edge-on from the Earth line of sight.

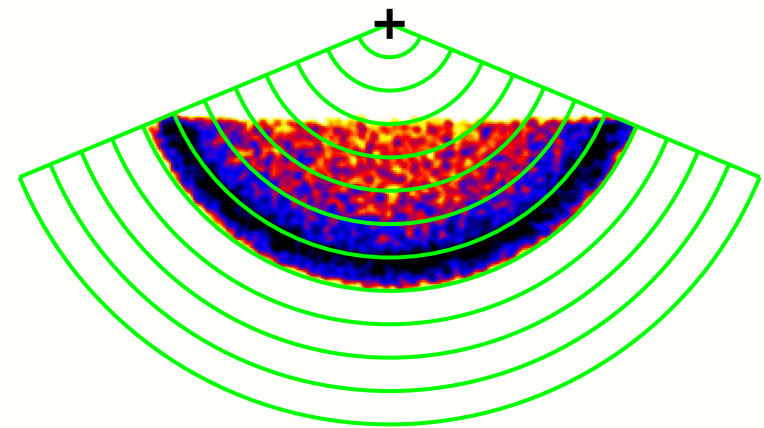

FIG. 4.- Smoothed projected counts from Zone 8 extending $49-56$ pc from the pulsar. The $6^{\prime}$ width spectral extraction regions are overlaid, demonstrating the overlap of counts on the interior regions. The location of the pulsar is marked with a cross.

the appropriate plane defined by the viewing angle. We then overlay the spectral extraction regions onto this projection and determine the fraction of counts which lie within each bin. Assuming each bin is spatially uniform, the fraction of counts within each region is equivalent to the fraction of flux. For all zones save the innermost, significant flux from each spatial zone is found in the interior spectral extraction regions, as demonstrated by Figure 4. Table 5 shows the fraction of flux from each zone which falls within each spectral region.

\subsection{Spatial Model}

Motivated by the morphology adopted in the previous section, we treat the PWN as a series of twelve expanding bubbles with the radial extent of each bubble accounted for by a preferential southerly expansion. As per the discussion in Section 3.3, this southerly expansion is assumed to result from an asymmetric reverse shock returning from the north. Particles are injected into each bubble for a period of time $\delta t_{i}$, after which the bubble is assumed to detach from the pulsar and direct injection of particles is discontinued. Given the distinct possibility that in the southerly direction SNR is still freely expanding (leading to nearly free expansion for the PWN), for simplicity we assume constant velocity expansion of the outer boundary of the nebula $v_{\text {outer }}=84 \mathrm{pc} / \mathrm{T}$, with $T$ the pulsar age determined by Equation 7. Despite the adoption of a constant outer expansion velocity, particles will still decelerate over time as they traverse the nebula and encounter supernova ejecta. In the interior of the nebula we therefore consider a radial velocity profile

$$
v(r, t)=v_{\text {outer }}\left(\frac{r}{R_{\text {outer }}}\right)^{\alpha}\left(\frac{t}{T}\right)^{-\alpha},
$$

with $\alpha$ a fit parameter, and the time dependence determined by the requirement that $v_{\text {outer }}$ remain constant. Integrating Equation 16 yields the position of various bubbles with time. The final extent of each bubble (e.g. Zone 1 covers $0-7$ pc from the pulsar) determines the required injection time for each bubble via Equation 13 .

Once injection ends, the bulk evolution of each bubble is primarily adiabatic, as IC cooling is relatively weak and the magnetic field is reduced to the point that synchrotron losses are severe only for the highest energy particles. For relativistic particles with adiabatic index $4 / 3$ the particle pressure is $P_{\text {pwn }}=E_{e} /\left(3 V_{\text {pwn }}\right)$, and for purely adiabatic expansion $P_{p w n} V_{p w n}^{4 / 3}=$ constant. For all time steps particle adiabatic losses are therefore computed via

$$
E_{e} \propto V_{p w n}^{-1 / 3}
$$

The sound speed in the relativistic gas of the PWN $(c / \sqrt{3})$ is far greater than the expansion velocity of the PWN, implying that pressure differences will rapidly equilibrate and that the PWN is nearly isobaric. The energy lost due to adiabatic expansion goes into accelerating supernova ejecta at the PWN-SNR interface, and so we consider adiabatic losses to be shared equally among all particles present in the nebula, with the fraction of energy lost by each particle a function only of the volume evolution of the aggregate nebula.

\subsection{Magnetic Field Model}

In principle, one can use the injected magnetic field energy (Equation 10) and spatial evolution of the PWN zones to compute the magnetic field $\left(E_{B}=\frac{B^{2}}{8 \pi} V\right.$, see Gelfand et al. (2009)). Due to the uncertainty in how the magnetic field is distributed and transported, and the possibility of field amplification in the post-shock flow, we adopt a somewhat simpler magnetic field evolution.

A static and uniform magnetic field presents the simplest possible magnetic field structure, yet the nebular magnetic field is expected to decrease from the termination shock to the far reaches of the nebula, as well as decrease with time as the pulsar spins down. Near the pulsar one common simple formulation is a dipolar magnetic field $\left(B \propto r^{-3}\right)$ out to the pulsar light cylinder, with a toroidal field $\left(B \propto r^{-1}\right)$ out to the termination shock. The surface magnetic field is estimated from the period and period derivative as

$$
B_{s}=3.2 \times 10^{19}(P \dot{P})^{1 / 2} \mathrm{G} .
$$

For PSR J1826-1334 this gives a current value of $B_{s}=2.8 \times$ $10^{12} \mathrm{G}$ for a surface radius $r_{s}=10 \mathrm{~km}$. The light cylinder is currently located at $r_{l c}=c / \Omega=4.8 \times 10^{8} \mathrm{~cm}$. At the wind termination shock the field is amplified by a factor of $\sim 3$, which gives $B_{t s} \approx 400 \mu \mathrm{G}$ for $r_{t s}=0.03 \mathrm{pc}$, and the assumed geometry of $B \propto r^{-3}$ out to the light cylinder and $B \propto r^{-1}$ from the light cylinder to the termination shock. 
TABLE 5

Projection of Spatial Zones onto Spectral Extraction Regions

\begin{tabular}{|c|c|c|c|c|c|c|c|c|c|c|c|c|c|}
\hline & Reg 1 & $\operatorname{Reg} 2$ & $\operatorname{Reg} 3$ & $\operatorname{Reg} 4$ & Reg 5 & Reg 6 & $\operatorname{Reg} 7$ & Reg 8 & Reg 9 & Reg 10 & $\operatorname{Reg} 11$ & $\operatorname{Reg} 12$ & $\mathrm{~V}\left(\mathrm{pc}^{3}\right)$ \\
\hline Zone 1 & 1.00 & & & & & & & & & & & & 440 \\
\hline Zone 2 & 0.14 & 0.86 & & & & & & & & & & & 3100 \\
\hline Zone 3 & 0.01 & 0.30 & 0.69 & & & & & & & & & & 4800 \\
\hline Zone 4 & & 0.07 & 0.34 & 0.59 & & & & & & & & & 16000 \\
\hline Zone 5 & & 0.01 & 0.13 & 0.34 & 0.52 & & & & & & & & 27000 \\
\hline Zone 6 & & & 0.05 & 0.15 & 0.32 & 0.47 & & & & & & & 40000 \\
\hline Zone 7 & & & 0.02 & 0.08 & 0.16 & 0.30 & 0.44 & & & & & & 56000 \\
\hline Zone 8 & & & & 0.04 & 0.09 & 0.16 & 0.30 & 0.41 & & & & & 75000 \\
\hline Zone 9 & & & & 0.02 & 0.06 & 0.10 & 0.15 & 0.28 & 0.39 & & & & 96000 \\
\hline Zone 10 & & & & 0.01 & 0.04 & 0.07 & 0.10 & 0.15 & 0.27 & 0.37 & & & 120000 \\
\hline Zone 11 & & & & & 0.02 & 0.05 & 0.07 & 0.10 & 0.15 & 0.26 & 0.35 & & 150000 \\
\hline Zone 12 & & & & & 0.01 & 0.03 & 0.05 & 0.07 & 0.10 & 0.15 & 0.25 & 0.34 & 180000 \\
\hline
\end{tabular}

NoTE. - Each row gives the fraction of flux from each spatial zone falling within each spectral region. For example, Column 1 , Row 2 indicates the fraction of flux from Zone 2 which falls within Region 1 (0.141). The flux within Region N is the sum of the fluxes in zones 1-12 times the coefficients $\left(C_{i, N}\right)$ in column $\mathrm{N}, F_{N}=\sum_{i=1}^{12} C_{i, N} F_{i}$.

The termination shock occurs at radius:

$$
r_{t s}=\left(\frac{\dot{E} \epsilon}{4 \pi c P_{p w n}}\right)^{\frac{1}{2}}
$$

where $\epsilon$ is the filling factor of the pulsar wind. For an isotropic wind $\epsilon=1$, though more common is a scaling with co-latitude $\theta$ of $\sin ^{2} \theta$, yielding $\epsilon=3 / 2$ (Bogovalov \& Khangoulyan 2002). The key feature here is that the termination shock radius scales as $r_{t s} \propto$ $\left(\dot{E} / P_{\text {pwn }}\right)^{1 / 2}$. One can estimate the PWN pressure as $P_{p w n} \propto \rho_{e j} v^{2}$ (Equation 26), with $\rho_{e j} \propto t^{-3}$ (Equation $24)$. The assumed constant velocity expansion therefore gives $P_{p w n} \propto t^{-3}$, which is identical to the $P_{p w n} \propto t^{-3.0}$ scaling computed by the Gelfand et al. (2009) model prior to reverse shock interactions. The termination shock radius accordingly scales roughly as $r_{t s} \propto \dot{E}(t)^{1 / 2} t^{3 / 2}$, and for a braking index near $n=2$ (where $\dot{E}(t) \propto t^{-3}$ ) the termination shock remains approximately static. Furthermore, since our SED model focuses on emission from the entire nebula (which dwarfs $r_{t s}$ ), the precise value of $r_{t s}$ has little effect on the resultant SED. For simplicity, we therefore assume a constant value of $r_{t s}=0.03 \mathrm{pc}$.

Adopting a dipolar followed by toroidal magnetic field geometry out to the termination shock, $B_{t s}=3 B_{l c} r_{l c} r_{t s}^{-1}=$ $3 B_{s} r_{s}^{3} r_{l c}^{-2} r_{t s}^{-1} \propto P^{-3 / 2} \dot{P}^{1 / 2} \propto \dot{E}(t)^{1 / 2}$. Therefore $B_{t s} \propto$ $\dot{E}(t)^{1 / 2}$ was much higher at earlier times when the pulsar spin-down was greatest. Past the termination shock the field continues to decrease, though likely at a slower rate than $B \propto 1 / r$, since this would put the field at $\ll 1 \mu \mathrm{G}$ in the outermost reaches of the nebula. Instead, we adopt a behavior of:

$$
B(r, t)=400\left(\frac{r}{0.03 \mathrm{pc}}\right)^{\beta}\left(\frac{\dot{E}(t)}{2.8 \times 10^{36}}\right)^{1 / 2} \mu \mathrm{G}
$$

past the termination shock, where $\beta$ is a fit parameter and the $400 \mu \mathrm{G}$ coefficient is the approximate value at the termination shock. We should emphasize that this magnetic field model does not conserve magnetic flux, since we assume that some magnetic field may be generated in the post-shock flow. The mean magnetic field within the boundaries of each zone is determined via a volume integration of Equation 20, and this is the value used at each time step since each zone is treated as homogeneous in the SED code.

\subsection{Diffusion}

A certain fraction of the injected particles will be lost from the nebula due to diffusive escape. For each zone we compute the diffusive flux between zones by solving:

$$
J_{i}\left(E_{e}, t\right)=-D \frac{\partial n_{i}\left(E_{e}, t\right)}{\partial R} \mathrm{~cm}^{-2} \mathrm{~s}^{-1}
$$

with $J_{i}\left(E_{e}, t\right)$ the diffusive flux in particles per $\mathrm{cm}^{2} \mathrm{~s}^{-1}$ for zone $i, n_{i}\left(E_{e}, t\right)$, the particle density, and $D$ the diffusion coefficient. The concentration gradient $\partial n_{i}\left(E_{e}, t\right) / \partial R$ is evaluated as the concentration difference between adjacent zones divided by the distance between zone midpoints. Solving Equation 21 for each zone at every time step gives the number of particles diffusing between zones. Hence, time permitting particles typically diffuse sequentially from inner zones to outer zones, stepping from Zone 1 to Zone 12. The PWNSNR interface will likely trap the majority of high-energy particles within the PWN. In addition, the surrounding SNR is expected to contain significant numbers of high energy particles. We therefore expect that particles will diffuse rather slowly out of the PWN and into the SNR, and so we arbitrarily set the density in the surrounding $\operatorname{SNR} n_{S N R}\left(E_{e}, t\right)=$ $0.8 \times n_{12}\left(E_{e}, t\right)$, which allows particles to slowly escape the PWN from Zone 12. The final SED depends very weakly on $n_{S N R}\left(E_{e}, t\right)$, however, given the high degree of cooling and large final size of the nebula.

Toroidal magnetic field lines tend to contain particles very effectively, and even with relatively rapid Bohm diffusion $\tau_{\text {esc }} \approx 40,000(R / 10 \mathrm{pc})^{2}\left(\mathrm{E}_{\mathrm{e}} / 100 \mathrm{TeV}\right)^{-1}(\mathrm{~B} / 10 \mu \mathrm{G})$ year (de Jager \& Diannati-Atai 2008). In the Bohm limit the crossfield mean free path $\lambda$ equals the particle gyroradius, which is exceedingly small: $\lambda=0.001\left(E_{e} / 100 \mathrm{TeV}\right)(\mathrm{B} / 10 \mu \mathrm{G})^{-1}$ parsec. If the field line structures contain radial components, however, particles diffuse far more rapidly. Turbulence and mixing caused by the passage of the reverse shock might provide the necessary disruption to the magnetic field structure to achieve radial field components. Even in young PWNe where significant reverse shock interaction has likely not yet occurred, however, the simple model of purely toroidal magnetic field has been challenged by comparing X-ray observations of PWNe with the predictions of the Kennel \& Coroniti (1984b) model. Chandra observations of 3C 58 (Slane et al. 2004) allowed comparison of the radial X-ray spectral index variation with the predictions of Kennel \& Coroniti (1984b). For any reasonable values of $\sigma$ these authors found the observed pho- 
ton index increased much slower in 3C 58 than expected from the model of Kennel \& Coroniti (1984b). A similar deviation is observed for G21.5 - 0.9 (Slane et al. 2000), implying that the magnetic field structure deviates from a purely toroidal structure.

The characteristic time scale for particle diffusion is determined by both the size of the nebula as well as diffusion coefficient $D$, such that $\tau_{e s c} \approx R^{2} / 6 D$. Alternately, the mean free path of a particle $\lambda \equiv 3 D / c$. The electron diffusion coefficient is frequently parameterized in power-law form $D\left(E_{e}\right)=D_{0}\left(E_{e} / E_{e, 0}\right)^{\delta}$. For Bohm diffusion $\delta=1$, while energy independent diffusion of course corresponds to $\delta=0$. To account for diffusion in a non-toroidal magnetic field we select a Bohm-type diffusion with $\delta=1$, such that mean free path and escape time scale as:

$$
\begin{aligned}
\lambda & =a E_{e} \\
\tau_{\text {esc }} & =R^{2}\left(2 a c E_{e}\right)^{-1}
\end{aligned}
$$

with $a$ left as a free parameter in the model such that the mean free path is allowed to vary from the Bohm value to account for complex nebular field structure. For simplicity we ignore any perturbation the magnetic field might have on the escape timescale, since clearly particles must primarily move along rather than across magnetic field lines.

\subsection{Photon Fields}

Common practice in PWN SED modeling has been to adjust the dust IR energy density to maximize the agreement between model and data. We instead fix the photon densities at published values, reducing the number of model fit parameters. Adopting three primary photon fields (CMBR, far IR (dust), and starlight), we employ the interstellar radiation mapcube within the GALPROP suite (Porter \& Strong 2005) to estimate the photon fields at the Galactic radius of PSR J1826-1334. A distance of $4 \mathrm{kpc}$ in the direction of PSR J1826-1334 corresponds to a Galactic radius of $4.7 \mathrm{kpc}$; at this radius, dust IR photons typically peak at $T \approx 32 \mathrm{~K}$ with a density of $\approx 1 \mathrm{eV} \mathrm{cm}^{-3}$, while stellar photons peak at $T \approx 2500 \mathrm{~K}$ with a density of $\approx 4 \mathrm{eV} \mathrm{cm}^{-3}$. At these densities IR and CMB photons contribute approximately equally to $\mathrm{TeV}$ inverse Compton emission, while stellar photons are inconsequential. Synchrotron self-Compton emission is negligible for the magnetic fields present in HESS J1825-137.

\subsection{SED Model}

A number of recent papers have applied various models to investigate the broadband emission from PWNe (Lemiere et al. (2009), Gelfand et al. (2009), Bucciantini et al. (2010), Fang \& Zhang (2010), Slane et al. (2010), Tanaka \& Takahara (2010)). Gelfand et al. (2009), in particular, constructed a detailed 1-D dynamical model of PWNe magnetic field, pressure, size, energetics and spectral evolution inside a host SNR. These authors demonstrated their model by fixing parameters to mimic the Crab Nebula. While this model certainly helps shed light on compact $\mathrm{PWNe}$ such as the Crab, the markedly different sizes of X-ray and $\mathrm{TeV}$ emission regions in HESS J1825-137 (along with most other middle aged Vela-like PWN) pose a challenge to 1-D models.

We therefore treat the nebula as a series of twelve expanding bubbles in order to match the twelve H.E.S.S. spatial regions. Each of these bubbles is treated as homogeneous, and

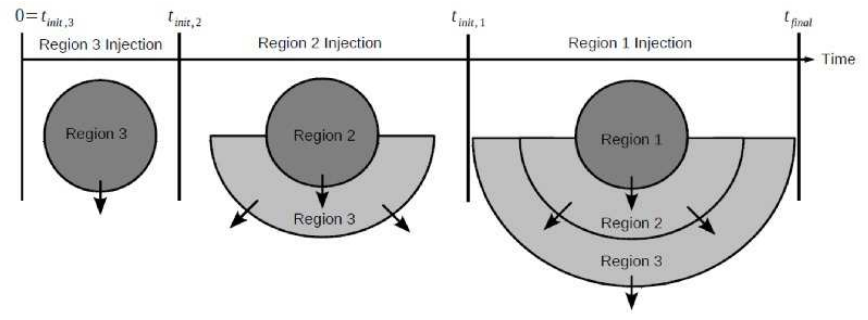

FIG. 5.- Snapshots of the evolution of nebular bubbles over three injection epochs. Dark gray corresponds to injection from the pulsar, light gray denotes the cooling phase, and arrows indicate diffusing particles.

filled with particles (assumed to be electrons) as well as magnetic field. We compute SEDs from evolving the electron populations over various lifetimes in a series of time steps, extending the single-zone approach taken in (Abdo et al. 2010), (Ackermann et al. 2011), (Grondin et al. 2011).

The twelve-zone modeling approach permits investigation of the twelve H.E.S.S. spectral extraction regions, yet the number of spatial zones need not be limited to the number of data regions and in theory one could utilize any number of zones and then project those zones onto the sky for SED construction. The spatial grid size is completely arbitrary, and hence the final SED plot should be independent of grid size. A finer grid of zones vastly increases computation time, however, and provides little new information given the limited number of data regions. Nevertheless, as a consistency check we double the number of zones (to 24) and plot the resultant SED. As expected, the model curves change imperceptibly in all but the innermost region. The slight alteration in Region 1 is due to the steep variation in the magnetic field near the pulsar. This lack of dependence of the SED on the spatial zone size provides a check of the SED model.

\subsection{Model Implementation}

Incorporating diffusion and projection effects between zones necessitates that all zones evolve in parallel, rather than independently. Evolution begins with Zone 12 at $t=t_{i, 12}=$ 0 , with escaping particles simply lost from the nebula. Once injection ends for Zone 12, at $t_{i, 11}$, Zone 11 begins to evolve, with its escaping particles injected into Zone 12. Once the injection period for Zone 11 ends, Zone 10 begins the injection phase, with Zones 11 and 12 in the cooling phases. This process is repeated until the current age of the pulsar is reached, with all twelve zones evolving simultaneously. Figure 5 shows a schematic of the evolution of the nebula for three spatial zones.

To compute the PWN SED, injected electrons are evolved through both the injection and cooling phases of each bubble's lifetime, with the age of each phase computed via Equation 13. Each zone shares the same injection spectrum, modulated by the spin down power. Cooling is computed from synchrotron (Blumenthal \& Gould 1970, Equation 4.5), inverse Compton (Blumenthal \& Gould 1970, Equation 2.56), and adiabatic losses (Equation 17). Evolution occurs in a series of time steps, such that during the injection phase at each time step we perform the following procedures:

1. Calculate the size of the zone by integrating Equation 16

2. Compute the mean magnetic field by integrating over the volume of the zone via Equation 20 
3. Inject pulsar wind particles into the zone according to Equation 10

4. Inject particles diffusing from adjacent zones according to Equation 21 (if including diffusion)

5. At each particle energy $E$, compute $\delta E$ from synchrotron, inverse Compton, and adiabatic losses

6. Calculate the subsequent particle spectrum at time $t+$ $\delta t: E(t+\delta t)=E-\delta E$

7. Remove escaping particles according to Equation 21 (if including diffusion)

Once injection ends, cooling is computed for the remainder of the evolution, following the same steps above (save for step 3 ). At each time step this process is repeated for all zones with $t_{i}>t$. When evolution completes, the synchrotron (Longair 2010, Equation 8.58), and IC (Blumenthal \& Gould 1970, Equation 2.48) fluxes are computed from the final electron spectrum and magnetic field for each zone. Finally, the flux within each region is tabulated using the coefficients of Table 5.

Model fitting is achieved by minimizing the $\chi^{2}$ between model and data using the downhill simplex method described in Press et al. (1992). Data consists of both X-ray and H.E.S.S. spectral flux points (77 in total): 12 in Region 1, 11 in Region 2, 9 in Region 3, and 5 data points for all exterior regions. For each ensemble of $N$ variable parameters we evolve the system over the pulsar lifetime and calculate $\chi^{2}$ between model curves and flux data points. The simplex routine subsequently varies the parameters of interest to minimize the fit statistic. We estimate parameter errors by computing $\chi^{2}$ for a sampling of points near the best fit values and using these points to fit the $N$-dimensional ellipsoid describing the surface of $\Delta \chi^{2}=2.71$. Under the assumption of Gaussian errors, the projected size of this $\Delta \chi^{2}=2.71$ ellipsoid onto each parameter axis defines the $90 \%$ multi-parameter (projected) error.

\section{RESULTS}

\subsection{Uniform Expansion, Constant Magnetic Field (Model 1)}

We begin by considering a very simple model of constant magnetic field $(\sim 3 \mu \mathrm{G})$, uniform expansion $(v(r, t)=$ constant), and ignore diffusive effects. Uniform expansion implies that each zone undergoes expansion for $1 / 12^{\text {th }}$ of the pulsar lifetime. Injection begins at pulsar birth $(t=0)$ for Zone 12, while Zone 1 begins evolution at $T-T / 12$, where $T$ is the current age of the pulsar. The large size of the PWN implies a relatively high age, and the braking index and $P_{0}$ selected in Table 6 give a total age of $21 \mathrm{kyr}$. This corresponds to a mean expansion velocity of $3900 \mathrm{~km} \mathrm{~s}^{-1}$. We initially adopt a minimum particle energy $E_{\text {min }}=100 \mathrm{GeV}$, and electron injection fraction $\eta=0.8$, which is a reasonable value for the expected particle dominated wind.

The model gives a poor match to the data, apparent in the SED plot of Figure 6. Applying the full fitting machinery to five variables (magnetic field $B$, initial spin period $P_{0}$, braking index $n$, electron power-law index $p$, electron cutoff $E_{c u t}$ ) yields an extremely low initial spin period $<1 \mathrm{~ms}$. Figure 6 therefore fixes the braking index $(n=3)$ and initial spin pe$\operatorname{riod}\left(P_{0}=15 \mathrm{~ms}\right)$ to reasonable values; the other three variables are fit and $90 \%$ multi-parameter error estimates are computed, as shown in Table 6 . The fit is still quite poor, and the

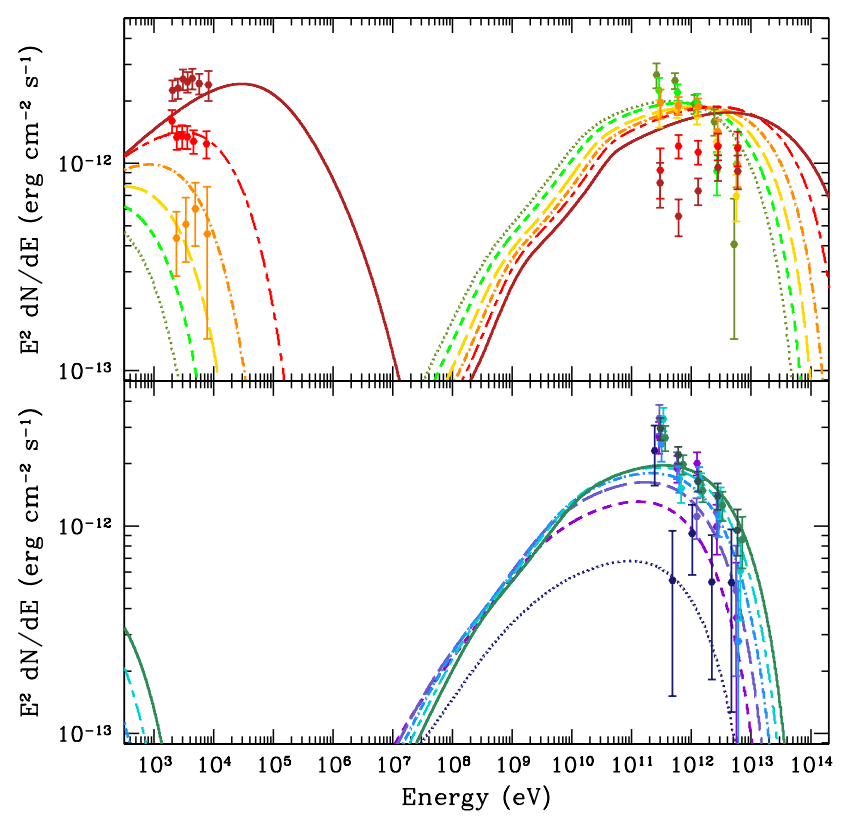

FIG. 6.- Model 1. Fitted simple spectral energy distribution model of the nebula with a constant magnetic field $(2.9 \mu \mathrm{G})$ throughout the nebula for all time, constant expansion velocity such that each zone undergoes injection for $1 / 12^{\text {th }}$ of the pulsar age $(21 \mathrm{kyr})$, and $\chi^{2} /$ d.o.f. $=575 / 74$. The SED plot is split into two panels for clarity, with a color scale evolving from dark red (Region 1, youngest), to green, to blue, to violet (Region 12, oldest). The line type evolves as well, from solid (inner, youngest) to long dot-dashed, short dot-dashed, long dashed, short dashed, and finally dotted (outer, oldest). The upper panel shows the model curves and H.E.S.S. data for the inner six regions, and Suzaku data for the inner three regions, while the lower panel shows the outer six regions. The fit is clearly quite poor, requiring fewer particles in the inner regions (and a higher magnetic field), with less cooling and more power in the outer regions. The gradual decrease of the cooling break energy with increasing region is primarily due to energy dependent synchrotron losses.

fit value of $E_{\text {cut }}=3200 \mathrm{TeV}$ is implausibly large. Inspection of Figure 6indicates that the normalization of each individual zone is highly skewed. The innermost zone has far too much VHE flux, while Zone 12 at the nebular frontier has far too little VHE flux.

\subsection{Velocity Profile, Constant Magnetic Field (Model 2)}

Though each region occupies the same $6^{\prime}$ width, the normalization issues of Figure 6 intimate that the injection time of the zones must vary with distance from the pulsar, and hence the flow speed in the nebula must not be constant. We therefore adopt a velocity profile in the nebula of $v(r, t)=$ $r^{\alpha} C(t)$ (Equation 16). The best fit with this model and six variables is shown in Table 7 . The radial velocity profile results in a lengthening of the injection phase with increasing radial distance from the pulsar, and the values $n$ and $P_{0}$ yield an age of 28 kyr. This model is shown in Figure 7, is far superior to Figure 6, and reproduces the VHE steepening trend moderately well for the inner six regions. Yet the outer model curves are all far steeper than the VHE data, implying an excess of synchrotron cooling for the $\sim 40 \mathrm{TeV}$ particles required to upscatter CMB photons to the $\sim 5 \mathrm{TeV}$ highest energy H.E.S.S. data point (Equation 4). As a result, in the outermost regions (presumably corresponding to the oldest electrons), electrons of energy $\sim 40 \mathrm{TeV}$ have almost completely burned off. In the innermost region the X-ray flux is signifi- 


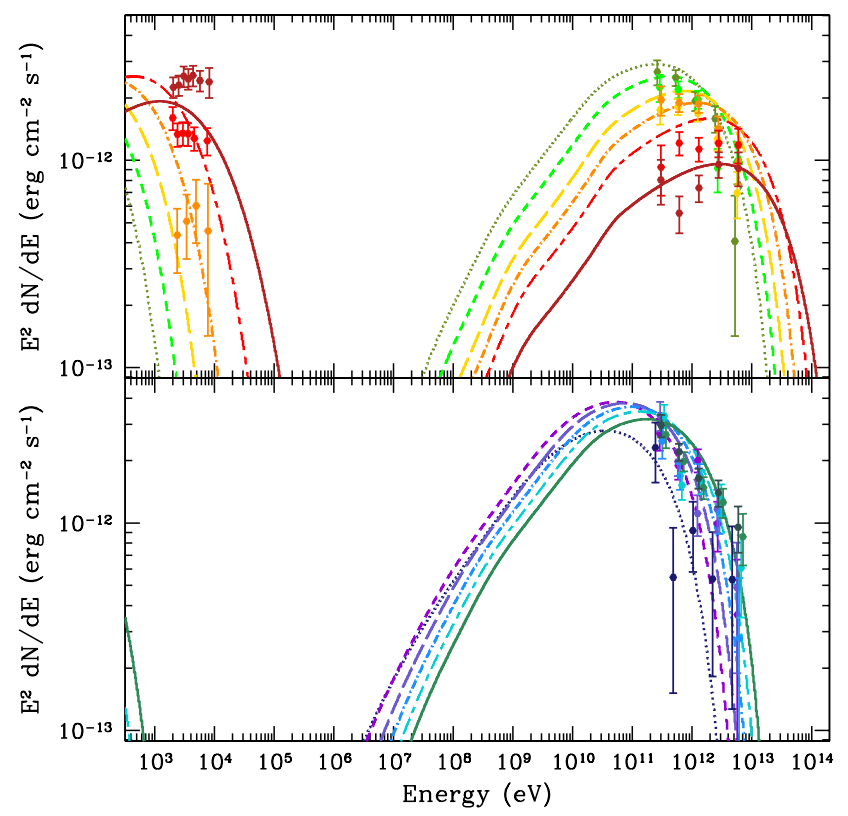

FIG. 7.- Model 2. Fitted SED model with a constant $5.6 \mu$ G magnetic field throughout the nebula for all time, $v(r) \propto r^{-0.36}$ such that the length of the injection phase increases with zone number, and $\chi^{2} /$ d.o.f. $=236 / 71$. The model curve line scheme is described in Figure 6 The radial velocity profile improves the fit over Figure 6 though the poor fit to the innermost X-ray data and excessive cooling in the outer zones suggest a radial magnetic field profile may provide a better fit.

cantly under-produced, suggesting the field must be $>5 \mu \mathrm{G}$ near the pulsar. Evidently a non-uniform magnetic field profile is required, with higher field in the interior to match X-ray fluxes and lower field in the exterior in order to reduce synchrotron cooling for the oldest electrons.

In order to better visualize the results of the SED fitting, we also show the ratios between the model zone fluxes and spectral indices and the measured data values (Figure 8). The model flux is computed by integrating the flux in the desired energy band (either $1-8 \mathrm{keV}$ or $0.25-10 \mathrm{TeV}$ ) and model indices are computed by fitting to a power-law in the appropriate band. The model fluxes and indices are compared to the data from Tables 3 and 4.

\subsection{Velocity Profile, Magnetic Field Profile (Model 3)}

In hopes of improving the fit of Figure 7 we invoke a radial magnetic field profile of the form $B(r, t) \propto r^{\beta} \dot{E}(t)^{1 / 2}$ (Equation 20), such that as particles traverse the nebula they experience an ever decreasing magnetic field. Similar to section 4.2, we select six fit parameters, though instead of fitting the final magnetic field value we fit the magnetic profile index $\beta$. The best fit model yields an age of $34 \mathrm{kyr}$, and Table 6 reveals an improved fit statistic over the constant magnetic field model. The non-constant magnetic field does a much better job of matching the innermost X-ray data (see Figures 9 and 10), though we still have difficulty matching the VHE indices in the outer (purple) zones.

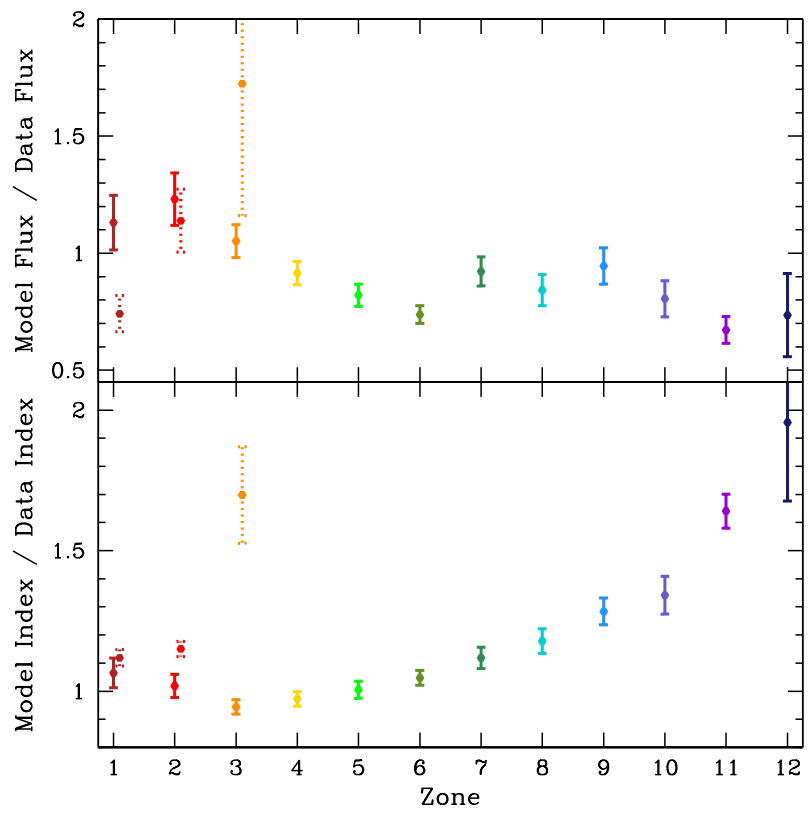

FIG. 8.- Model 2. Top: Ratio of model flux to the data listed in Tables 3 and 4, with X-ray $1-8 \mathrm{keV}$ flux ratios for the inner three zones (dotted) and VHE $0.25-10 \mathrm{TeV}$ flux ratios (solid) for all twelve zones. Bottom: Powerlaw indices over the same energy bands, with the model steadily worsening in the the outer zones.

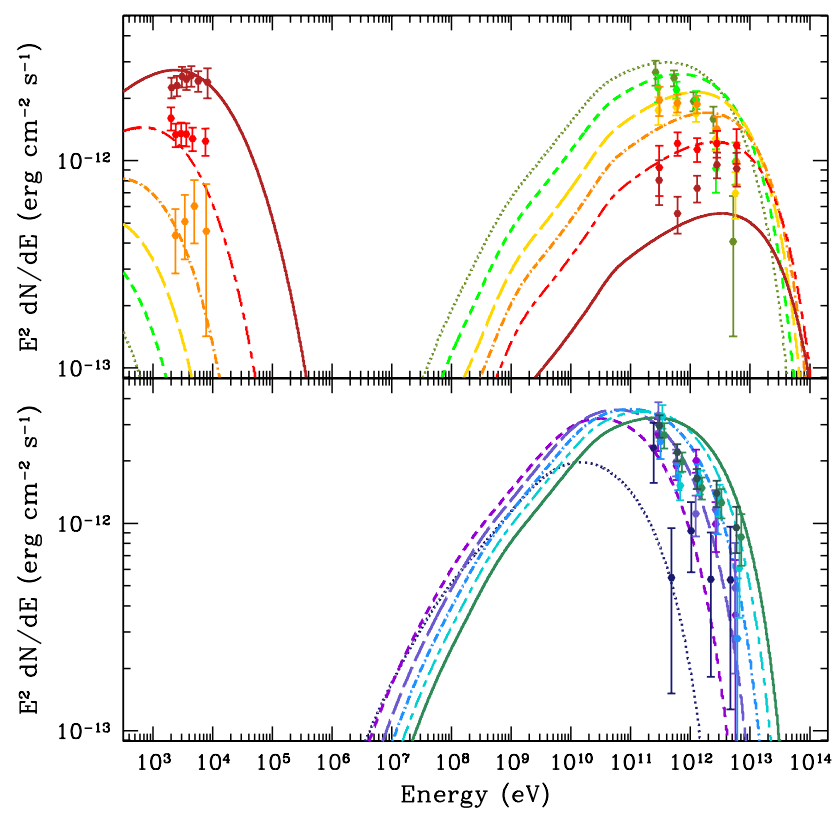

FIG. 9.- Model 3. Fitted SED model with $B(r, t) \propto r^{-0.74} \dot{E}(t)^{1 / 2}$, $v(r) \propto r^{-0.60}$, and $\chi^{2} /$ d.o.f. $=217 / 71$. The model curve line scheme is described in Figure 6 The radial magnetic field profile results in a superior fit to the innermost X-ray data compared to Figure 7 though we still suffer from excessive cooling losses in the outermost zones. 


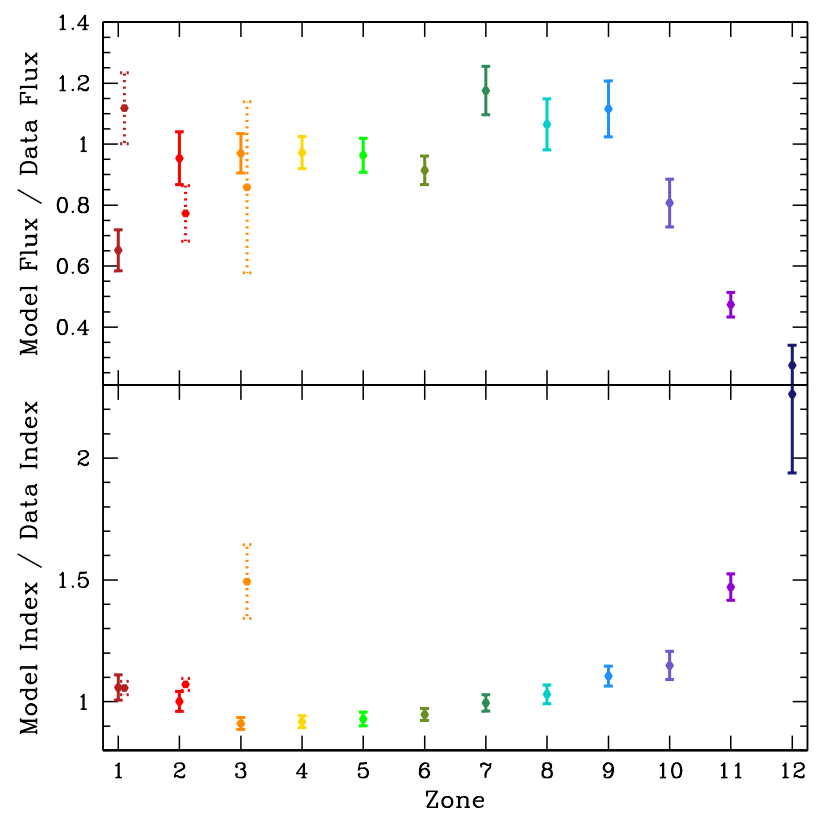

FIG. 10.- Model 3. Top: Ratio of model flux to the data listed in Tables 3 and 4, with X-ray $1-8 \mathrm{keV}$ flux ratios for the inner three zones (dotted) and VHE $0.25-10 \mathrm{TeV}$ flux ratios (solid) for all twelve zones. The X-ray flux ratios are much improved over Figure 8 Bottom: Power-law indices, with the model steadily worsening in the the outer zones.

\subsection{Velocity Profile, Magnetic Field Profile, Diffusion (Model 4)}

The severe under-prediction of VHE flux and overprediction of VHE index in the outer two zones of Figures 9 and 10 indicates a general lack of electrons with $E>10$ $\mathrm{TeV}$. One possibility of addressing this issue is simply reduced cooling by lowering the magnetic field. Yet even in Figures 7 and 8 with a low $6 \mu \mathrm{G}$ field the Region 12 model is far steeper than the data due to excessive cooling. In addition, Figures 9 and 10 demonstrate that higher fields are needed in the inner zones, which only serves to hasten synchrotron burn-off. Particle re-acceleration as particles traverse the nebula might explain the VHE flux $\sim 80 \mathrm{pc}$ from the pulsar, perhaps due to reverse shock interactions. Such acceleration is highly speculative, however, and beyond the scope of this paper. A more standard method of maintaining the high energy particle population far from the pulsar, which we explore below, is rapid energy-dependent diffusion with mean free path $\lambda \propto E_{e}$.

For this model we select seven variables: velocity radial profile index $\alpha$, magnetic profile index $\beta$, initial spin period $P_{0}$, braking index $n$, electron power-law index $p$, electron exponential cutoff $E_{c u t}$, and mean free path $\lambda$. Figure 11 shows the SED of the best fit model, scaled to display the aggregate gamma-ray data. This aggregate data is discussed in Section 5. Figure 12 displays the flux and index ratios for this model. The overall fit to the twelve spectral regions is far superior to previous models, particularly in the outer nebula. The largest departure of the model from the data is the X-ray spectral index of Region 3, though we remind the reader that the very hard Region 3 X-ray index reported in Table 4 may reflect residual background contamination. Table 6 lists the fit parameters, while Table 7 lists derived parameters from the fit (injection time, final magnetic field, and $\chi^{2}$ ). As expected from the scaling relations of section 3.1 , the particle residence time of Zone 1 is well under 1000 years. The braking index $n$ and initial spin period $P_{0}$ yield an age of $40 \pm 9 \mathrm{kyr}$ and a mean expansion velocity of $2000 \mathrm{~km} \mathrm{~s}^{-1}$. The mean free path of $\lambda=1.8\left(E_{e} / 100 \mathrm{TeV}\right)$ parsec predicts an escape timescale of $\tau_{\text {esc }} \approx 90(R / 10 \mathrm{pc})^{2}\left(\mathrm{E}_{\mathrm{e}} / 100 \mathrm{TeV}\right)^{-1}$ year. This model does have some difficulty matching the H.E.S.S. flux and slope in Region 1, which is due to the rapid diffusive loss of high energy particles. Most likely, the simple diffusive model employed vastly oversimplifies the complex nature of this source, and diffusion coefficients vary in both space and time. Nevertheless, the $\chi^{2} /$ d.o.f. for Model 4 indicates that the general fit is quite good.

As a check of our magnetic field model, we also allow the normalization at the termination shock to vary from its adopted value of $400 \mu \mathrm{G}$. The best fit model with this extra fit parameter yields a termination shock field of $320 \mu \mathrm{G}$ with no improvement of the fit statistic $\left(\chi^{2} /\right.$ d.o.f. $=118 / 69$, versus $\chi^{2} /$ d.o.f. $=119 / 70$ for the field fixed. Fitting the magnetic field normalization therefore does not yield a markedly better fit, and the termination shock value of $400 \mu \mathrm{G}$ appears quite reasonable. One further check of the magnetic field model is achieved by fitting a model with diffusion over uniform magnetic field (simply replacing the magnetic field radial index of Model 4 with a constant magnetic field value). This model cannot adequately match the data, yielding a best fit of $\chi^{2} /$ d.o.f. $=429 / 70$, and rules out uniform magnetic field as a viable option. The adopted IR photon density of $\approx 1$ $\mathrm{eV} \mathrm{cm} \mathrm{cm}^{-3}$ could easily vary by a factor of $\sim 2$, though varying the photon density does not qualitatively change the results presented here. A higher (lower) IR photon density means fewer (more) high energy electron are required to match the HESS data, implying a slightly softer (harder) electron spectral index or lower (higher) high energy cutoff.

\subsubsection{Relativistic Maxwellian Plus Power-Law Injection Spectrum}

The SED model described in the previous section takes into account only X-ray and VHE H.E.S.S. data, though ideally the model should be consistent with Fermi LAT data as well. Note that while we do not fit to the LAT points in Figure 11. the rough agreement between these data and the integrated (total) model flux in the LAT band is encouraging. However, the Fermi LAT photon index of $\Gamma=1.4 \pm 0.2$ (Grondin et al. 2011) predicts an electron index of $p=2 \Gamma-1=1.8 \pm 0.4$. This is somewhat harder than the electron index of $p=2.2$ utilized in Figures 11 and 12 , and an injection spectrum with fewer low energy electrons might plausibly improve the fit to the LAT data.

One option is adopting the relativistic Maxwellian plus power-law tail electron spectrum proposed by Spitkovsky (2008), and described in modeling terms by Grondin et al. (2011). Fitting with this model requires one additional parameter (the temperature of the Maxwellian), bringing the total up to eight. The best fit model with this injection spectrum yields a fit somewhat worse than the power-law case $\left(\chi^{2} /\right.$ d.o.f. $=123 / 69$, versus $122 / 70$ for the power-law spectrum). In addition, an extremely short initial spin period of $P_{0} \sim 2 \mathrm{~ms}$ is required, and the low $k T=0.2 \mathrm{TeV}$ results in a severe over-prediction of $\mathrm{GeV}$ flux and a far worse fit to the LAT data than the power-law case. Grondin et al. (2011) found an adequate fit to the aggregate data with a one-zone model, yet the inclusion of multiple zones seems to wash out any advantage of a relativistic Maxwellian electron spectrum. 
TABLE 6

Multiwavelength SED Fit To All Regions

\begin{tabular}{ccccccccc}
\hline \hline Model & $\alpha\left(v \propto r^{\alpha}\right)$ & $B / \beta\left(B \propto r^{\beta}\right)$ & $P_{0}(\mathrm{~ms})$ & $n\left(\dot{\Omega} \propto \Omega^{n}\right)$ & $p$ & $E_{c u t}(\mathrm{TeV})$ & $\lambda\left(\frac{E_{e}}{100 \mathrm{TeV}}\right) \mathrm{pc}$ & $\chi^{2} /$ d.o.f. \\
\hline 1 & - & $2.9 \pm 0.3^{\mathrm{a}}$ & $15^{\mathrm{b}}$ & $3^{\mathrm{b}}$ & $2.42 \pm 0.06$ & $3800 \pm 800$ & - \\
2 & $-0.36 \pm 0.03$ & $5.6 \pm 0.4^{\mathrm{a}}$ & $12 \pm 3$ & $2.5 \pm 0.1$ & $2.30 \pm 0.01$ & $130 \pm 10$ & - & $236 / 71$ \\
3 & $-0.60 \pm 0.08$ & $-0.74 \pm 0.02$ & $14 \pm 7$ & $2.2 \pm 0.1$ & $2.31 \pm 0.02$ & $190 \pm 30$ & - \\
4 & $-0.51 \pm 0.09$ & $-0.69 \pm 0.01$ & $13 \pm 7$ & $1.9 \pm 0.2$ & $2.24 \pm 0.02$ & $230 \pm 90$ & $1.8 \pm 0.3$ & $119 / 70$ \\
\hline
\end{tabular}

${ }^{a}$ Final (uniform) magnetic field $(\mu \mathrm{G})$

${ }^{b}$ Held fixed

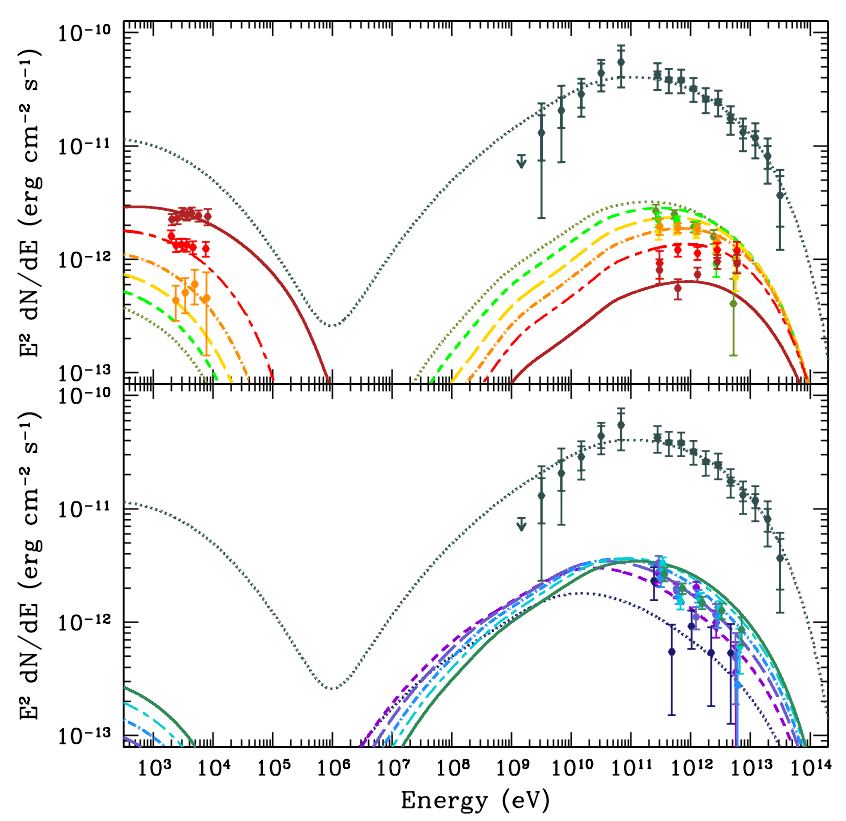

FIG. 11.- Model 4. Fitted SED model, scaled to show the aggregate data, with $B(r, t) \propto r^{-0.69} \dot{E}(t)^{1 / 2}, v(r) \propto r^{-0.51}, \lambda=1.8\left(E_{e} / 100 \mathrm{TeV}\right)$ parsec, and $\chi^{2} /$ d.o.f. $=119 / 70$. The model curve line scheme is described in Figure 6 The inclusion of rapid diffusion among zones provides enough high energy electrons in the outer nebula to match the H.E.S.S. data in Zones $10-12$. The slate data points denote the aggregate Fermi LAT and H.E.S.S data, with both statistical and systematic error estimates. The slate dotted line (representing the sum of flux from all twelve zones) is scaled up by $25 \%$ to account for the greater total flux in the aggregate extraction region (see Section 2.2). The overall fit to the aggregate data with this scaling is quite impressive.

TABLE 7

DERIVEd PARAMETERS FOR MODEl 4

\begin{tabular}{cccccc}
\hline \hline Zone & $R(\mathrm{pc})$ & $\delta t_{i}(\text { year })^{\mathrm{a}}$ & $B(\mu \mathrm{G})^{\mathrm{b}}$ & $\chi^{2}$ & \# data points \\
\hline 1 & 7 & 600 & 12 & 23 & 12 \\
2 & 14 & 1200 & 6.6 & 13 & 11 \\
3 & 21 & 1500 & 4.8 & 6.6 & 9 \\
4 & 28 & 1900 & 3.8 & 4.6 & 5 \\
5 & 35 & 2200 & 3.2 & 8.0 & 5 \\
6 & 42 & 2500 & 2.8 & 10 & 5 \\
7 & 49 & 2900 & 2.5 & 8.9 & 5 \\
8 & 56 & 3300 & 2.2 & 11 & 5 \\
9 & 63 & 3800 & 2.1 & 7.4 & 5 \\
10 & 70 & 4400 & 1.9 & 6.4 & 5 \\
11 & 77 & 5500 & 1.8 & 16 & 5 \\
12 & 84 & 9900 & 1.7 & 4.4 & 5 \\
\hline
\end{tabular}

\footnotetext{
${ }^{\mathrm{a}}$ Injection timescale
}

${ }^{\mathrm{b}}$ Final magnetic field

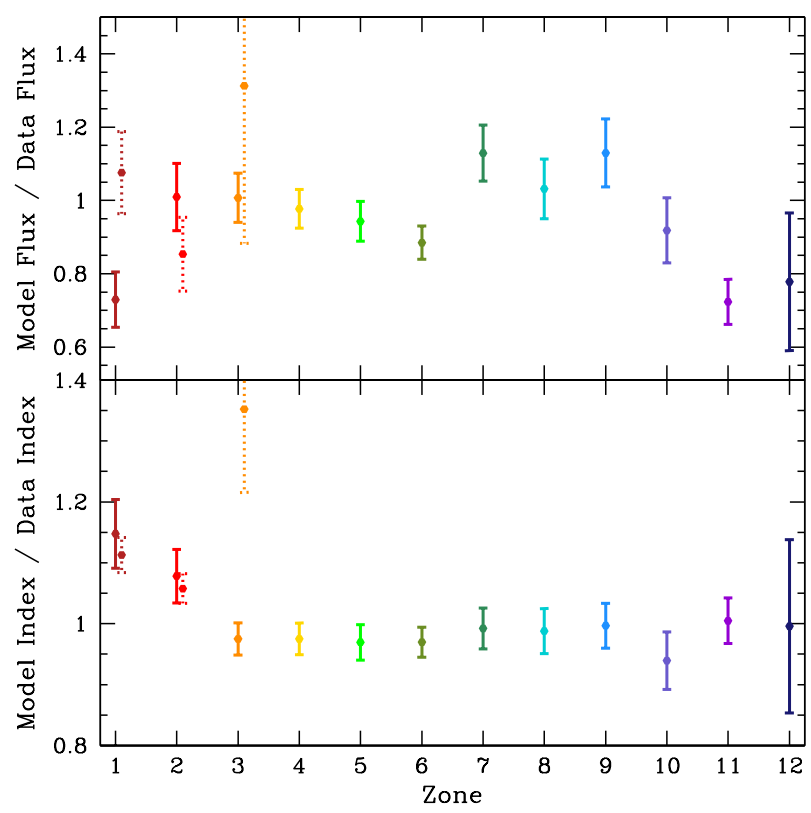

FIG. 12.- Model 4. Top: Ratio of model flux to the data listed in Tables 3 and 4, with X-ray $1-8 \mathrm{keV}$ flux ratios for the inner three zones (dotted) and VHE $0.25-10 \mathrm{TeV}$ flux ratios (solid) for all twelve zones. Bottom: Power-law indices. For both upper and lower panels the outer-zone ratios are far superior to Figures 8 and 10

\section{DISCUSSION}

As an initial check of the validity of the scalings applied to both the Suzaku and H.E.S.S. data, we refit Model 4 with the raw data. Using the original H.E.S.S. data points and X-ray data taken from regions coinciding with the HESS quarter annuli, the best fit parameters are within $1 \sigma$ of the values listed in Table 6 for Model 4, except for the magnetic field index. The different H.E.S.S. flux ratios between interior zones results in a magnetic field index that differs from the Model 4 value by +0.02 , or $2 \sigma$. Despite the similarity in fit parameters, however, the fit statistic increases dramatically, from $\chi^{2} /$ d.o.f. $=119 / 70$ for Model 4, to $\chi^{2} /$ d.o.f. $=169 / 70$. Nearly all of this increase in $\chi^{2}$ comes from the inner two regions. The H.E.S.S. excess map (Figure 1b) reveals that there appears to be significantly more flux $6^{\prime}-12^{\prime}$ from the pulsar than within $6^{\prime}$ of the pulsar. Yet due to the small original H.E.S.S. extraction region for the $6^{\prime}-12^{\prime}$ region (which is $25 \%$ smaller than the $0-6^{\prime}$ region), the reported H.E.S.S. fluxes are identical for the inner two regions. The use of identical fluxes for the inner two zones therefore results in a worse fit for the model, which assumes radial variations in the flow speed and a greater number of particles farther from the pul- 
sar. Nevertheless, the commonality of the fit parameters with the raw and scaled data provides some support for the scaled approach taken throughout this paper. One further check of the X-ray scaling is to fit the scaled H.E.S.S. data and raw, unscaled X-ray data. With this slightly lower X-ray flux the best fit parameters are well within errors for Model 4, and give a nearly identical $\chi^{2}$.

Of the four models scenarios described above, the final model which incorporates diffusion provides by the far the best fit to the data. The relativistic Maxwellian injection spectrum yields a somewhat worse fit statistic to the simple powerlaw case, and though the power-law injection spectrum also better matches the Fermi LAT data, definitive discrimination between lepton injection spectra is not possible at this stage. Yet we can convincingly argue that the pulsar wind must inject significant numbers of electrons up to energies of at least $100 \mathrm{TeV}$, the low energy power-law index must be $p \sim 2$ in order to match the Fermi LAT data, that a high percentage of the pulsar spin-down energy must go into leptons, and that some mechanism (we invoke diffusion) maintains high energy particles far from the pulsar. In the following discussion we consider the simple power-law spectrum injection spectrum (Model 4) shown in Figures 11 and 12

The sum of fluxes from the twelve spatial zones has been scaled up some $25 \%$ in Figure 11 due to the fact that our spectral extraction regions do not capture the entirety of the counts within the aggregate nebula. This scaled flux ideally should match the Fermi LAT and H.E.S.S. aggregate data points (which both represent larger spatial regions), which appears to be the case for Figure 11 Computing the fit statistic for the aggregate H.E.S.S. and LAT data gives $\chi^{2}=27$ for the 16 gamma-ray data points (assuming only statistical errors on the data points, and ignoring the LAT upper limit). Given the very small statistical errors on the H.E.S.S. data points the agreement between gamma-ray data and the summed model curves is remarkable. For comparison, the best fit model utilizing the relativistic Maxwellian injection spectrum nets a $\chi^{2}=50$ for the 16 gamma-ray data points. The 'missing' $\approx 25 \%$ of flux implies a missing population of particles contributing to the IC flux of the nebula. Such particles have likely diffused to the north and east of the pulsar exterior to our extraction regions. A tweak of the initial pulsar spin-down from $13 \mathrm{~ms}$ to $11 \mathrm{~ms}$ (well within errors) provides $\approx 40 \%$ greater spin-down energy, more than enough to enough to raise the model bulk nebular flux to the observed level.

The models proposed above utilize $\eta=0.8$, and we find an adequate fit to the X-ray and H.E.S.S. data for $P_{0}=13 \mathrm{~ms}$. Neglecting the 'missing' energy component, this connotes a total of $1.1 \times 10^{50} \mathrm{erg}$ of pulsar spin-down energy. Disregarding losses, we therefore expect of order $10^{49} \mathrm{erg}$ in magnetic field energy. While the magnetic field evolution and profile adopted in our model does not take into account the total energy budget of the pulsar, a reasonable model should nevertheless contain $\sim 10^{49}$ erg of magnetic energy for the choice of $\eta=0.8$. Computing the magnetic field energy from the final magnetic field profile $B(r, T)=400(r / 0.03 \mathrm{pc})^{-0.69} \mu \mathrm{G}$ yields an energy of $4.4 \times 10^{48} \mathrm{erg}$, which is quite reasonable given that the magnetic field likely experiences some losses due to expansion. Even though the vast majority of the pulsar energy initially goes into particles, summing the energy content of the final electron spectrum reveals that electron cooling losses leave only $6.9 \times 10^{48}$ erg remaining in the form of leptons. We can also compute the value of $\sigma$ from the assumed termination shock radius of $0.03 \mathrm{pc}$ and $400 \mu \mathrm{G}$ field strength. Adopting a flow speed at the termination shock of $c / 3$ and a spherical geometry, one achieves an estimate of $7 \times 10^{35} \mathrm{erg} \mathrm{s}^{-1}$ of magnetic energy flux at the termination shock. For the pulsar spindown value of $\dot{E}=2.8 \times 10^{36} \mathrm{erg} \mathrm{s}^{-1}$ this yields $\sigma \approx 0.3$. This value is consistent with the adopted value of $\eta=0.8$, which corresponds to a value of $\sigma=(1-\eta) / \eta=0.25$. One further consistency check for our model comprises comparing the available spin-down power to the total energy present in the nebula plus cooling and escape losses. Indeed, summing the leptonic energy, magnetic energy, cooling losses, and escape losses yields $1.1 \times 10^{50} \mathrm{erg}$, precisely the energy available from the pulsar.

The energy lost due to adiabatic cooling goes into accelerating a shell of swept-up material, and we can use estimates of this swept-up mass as another check of the validity of the SED model. For the adopted SNR density profile of a constant density inner core surrounded by a $\rho \propto r^{-9}$ outer envelope the transition between the two density regimes propagates out at a constant velocity $v_{t}$, such that (Blondin et al.2001):

$$
v_{t} \approx 6500\left(\frac{E_{S N}}{3 \times 10^{51} \mathrm{erg}}\right)^{1 / 2}\left(\frac{M_{e j}}{8 M_{\odot}}\right)^{-1 / 2} \mathrm{~km} \mathrm{~s}^{-1} \text {.(23) }
$$

This is much faster than the Model 4 outer PWN expansion of $2000 \mathrm{kms}^{-1}$, so we can treat the PWN as always expanding into the constant density ejecta core, with density Blondin et al. (2001):

$$
\begin{aligned}
\rho_{e j}(t) \approx & 3.7 \times 10^{-29}\left(\frac{E_{S N}}{3 \times 10^{51} \mathrm{erg}}\right)^{-3 / 2}\left(\frac{M_{e j}}{8 M_{\odot}}\right)^{5 / 2} \\
& \times\left(\frac{t}{20 \mathrm{kyr}}\right)^{-3} \mathrm{~g} \mathrm{~cm}^{-3} .
\end{aligned}
$$

The amount of mass swept up by the PWN over its lifetime can be approximated as:

$$
M_{s w}=\int_{t_{i}}^{T} \rho_{e j}(t) \zeta 4 \pi r^{2} d r
$$

with $t_{i}$ the initial timestep, $r=v_{\text {outer }} t$ and $\zeta$ the filling factor of the PWN, equal to 0.31 for the adopted morphology. Integrating Equation 25 over the $40 \mathrm{kyr}$ lifetime of the pulsar provides a very rough estimate of $1 M_{\odot}$ of swept up mass. Accelerating $1 M_{\odot}$ to $2000 \mathrm{~km} \mathrm{~s}^{-1}$ (the mean expansion velocity for a pulsar age of $40 \mathrm{kyr}$ ) requires some $4 \times 10^{49} \mathrm{erg}$. For the parameters selected in Model 4, the SED model posits a total adiabatic loss of $3.4 \times 10^{49} \mathrm{erg}$, quite close to the value quoted above. Recall that in the SED model adiabatic losses depend only on the assumed PWN size evolution (Equation 17), and do not take into account any SNR properties. Therefore the fact that the two adiabatic loss estimates are not glaringly different provides some support to the soundness of the SED model.

The computed pressure within the entire nebula can be used as a further sanity check on our model by comparing to the expected PWN pressure after $40 \mathrm{kyr}$. Given the degree of particle cooling, the model's final particle pressure $\left(P_{p w n}=\frac{E_{p}}{3 V_{p w n}}=1.0 \times 10^{-13} \mathrm{erg} \mathrm{cm}^{-3}\right)$ is less than the magnetic pressure $\left(\frac{B^{2}}{8 \pi}=1.9 \times 10^{-13} \mathrm{erg} \mathrm{cm}^{-3}\right)$. Within a freely expanding SNR the PWN pressure scales as 
(van der Swaluw et al. 2001):

$$
P_{p w n} \approx 3 / 25 \rho_{e j}(t) v_{p w n}^{2},
$$

with $\rho_{e j}(t)$ given by Equation 24, and $v_{p w n}$ the PWN expansion velocity. After $40 \mathrm{kyr}$ this predicts a mean nebular pressure of $P_{\text {pwn }} \approx 2 \times 10^{-13} \mathrm{erg} \mathrm{cm}^{-3}$, in good agreement with the computed values.

The adopted termination shock radius of $0.03 \mathrm{pc}$ predicts a pressure of $\approx 1 \times 10^{-9} \mathrm{erg} \mathrm{cm}^{-3}$ at the termination shock (Equation 19), significantly higher than the mean pressure of $\approx 3 \times 10^{-13} \mathrm{erg} \mathrm{cm}^{-3}$. This high pressure at the shock rapidly dissipates away from the pulsar, however, with the bulk of the nebula nearly isobaric at late times; each of the 10 outer zones has a pressure within $\approx 50 \%$ of the mean nebular pressure, as evidenced in the pressure and energy plot of Figure 13 This plot illustrates that the magnetic pressure exceeds particle pressure for the inner regions, and that the total particle energy exceeds the bulk magnetic field energy in the nebula. The total magnetic field pressure scales as $P_{p w n, B} \propto t^{-3.0}$, while the total particle pressure evolves as $P_{p w n, p} \propto t^{-2.9}$. This is precisely the $P_{\text {pwn }} \propto t^{-3}$ evolution predicted by Equations 24 and 26 for a freely expanding PWN.

In addition to the PWN pressure and energy we can also compute a variety of quantities which vary over the pulsar lifetime. We calculate the mean magnetic field evolution as $\bar{B} \propto t^{-1.6}$, which is quite similar to the $B \propto t^{-1.7}$ evolution computed by Gelfand et al. (2009) in their single-zone model during the free expansion phase. The various luminosities in the nebula are plotted in Figure 14 revealing that at late times the total luminosity of the nebula exceeds the pulsar spindown power. This figure also demonstrates that after a few thousand years adiabatic losses overtake synchrotron radiation to provide the dominant cooling mechanism, though particles of energy $E_{e}>10 \mathrm{TeV}$ primarily lose energy from synchrotron and IC cooling since for both the cooling timescale $\tau \propto E_{e}^{-1}$. Indeed, at late time the IC cooling actually exceeds the synchrotron cooling due to the low mean magnetic field of $\approx 2 \mu \mathrm{G}$.

We construct radial flux profiles by summing the model fluxes in the Suzaku and H.E.S.S. wavebands within each region, which can be compared with the data points plotted in Figure 2. We are also free to compute the profile in any arbitrary energy range, and so in Figure 15 we plot the projected surface brightness in the Fermi LAT 1-100 GeV range. Since the LAT energy range lies in the uncooled regime, the flux primarily tracks the energy content of electrons. As a result, the LAT flux peaks toward the outer realm of the nebula where the majority of electrons were injected when the pulsar was much younger with a far higher $\dot{E}$, and this gives that a rather uniform counts map in the LAT energy band.

Comparison of point source and extended Gaussian morphologies in the $10-100 \mathrm{GeV}$ range by Grondin et al. (2011) yielded a significance of $\approx 8.5 \sigma$ for a source extension of $0.56^{\circ} \pm 0.07^{\circ}$ centered $0.5^{\circ}$ southwest of the pulsar. Similar results are achieved with a uniform disk morphology, though the extension increases to $0.67^{\circ} \pm 0.02^{\circ}$. Fitting the source to a spatial template provided by the H.E.S.S. excess map $(E>200 \mathrm{GeV})$ decreases the test statistic by $42 \approx 6.5 \sigma$, implying that the LAT emission shows a distinctly different morphology from the H.E.S.S. emission. Indeed, the LAT source extension of $\sim 0.6^{\circ}$ centered $0.5^{\circ}$ southwest of the pulsar is remarkably consistent with Figure 15 .

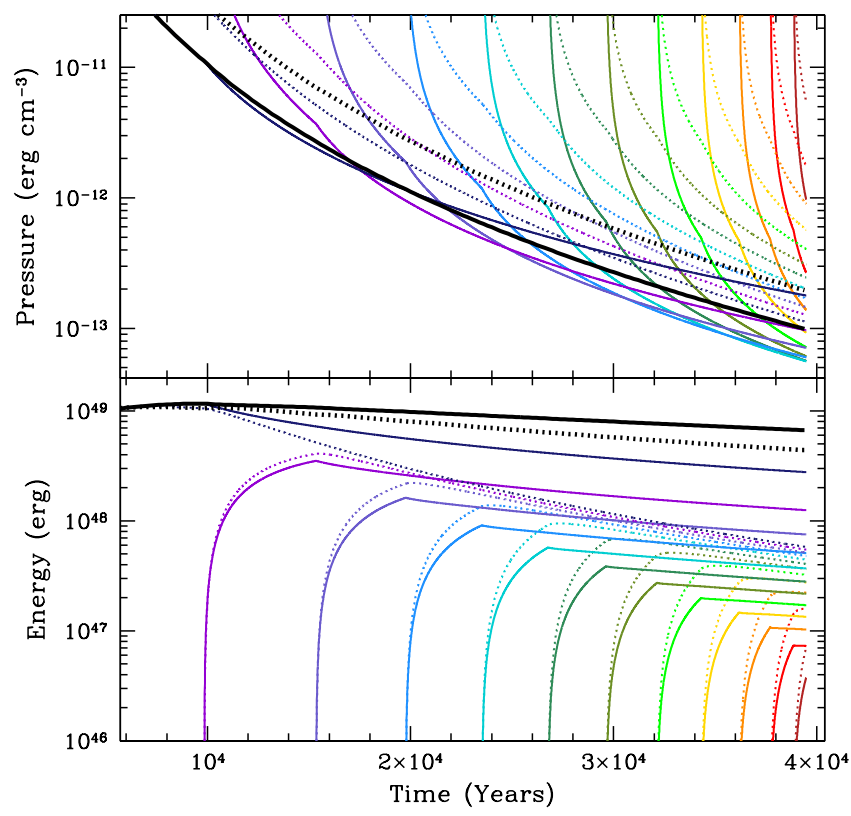

FIG. 13.- Top: Magnetic field pressure (dotted) and particle pressure (solid) of each zone. Total pressures are marked by the thick black lines. Bottom: Magnetic field energy (dotted) and particle energy (solid) of each zone. Black lines mark total energies. In both panels the values correspond to Model 4.

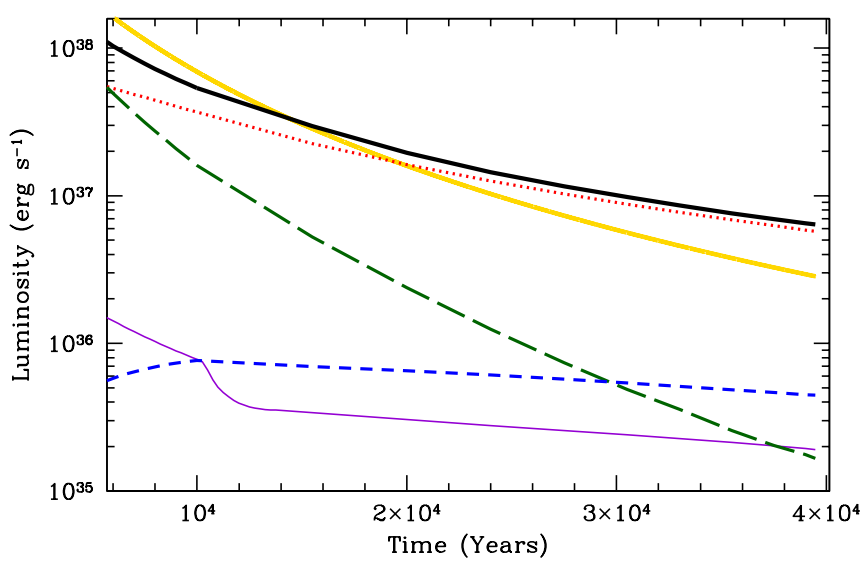

FIG. 14.- Bulk luminosity of the nebula for Model 4. The solid gold line denotes pulsar spin-down, while the solid black line shows the total luminosity from all processes. Synchrotron (green long-dashed), IC (blue shortdashed), and adiabatic (red dotted) cooling is also shown. The violet line denotes diffusive escape losses from the nebula.

\section{CONCLUSIONS}

Analysis of recently public Suzaku X-ray data covering the majority of the very bright VHE source HESS J1825-137 indicate that $\mathrm{X}$-rays extend only $\approx 1 / 4$ as far as VHE gammarays. The variable extent of the nebula in different wavebands, as well as the observed VHE spectral softening away from the pulsar, is naturally explained by electron cooling losses. Time-dependent 3-D modeling of the twelve data regions allows one to constrain the electron injection properties, spin-down behavior of the pulsar, nebular velocity profile, and magnetic field profile. A choice of $80 \%$ of the 


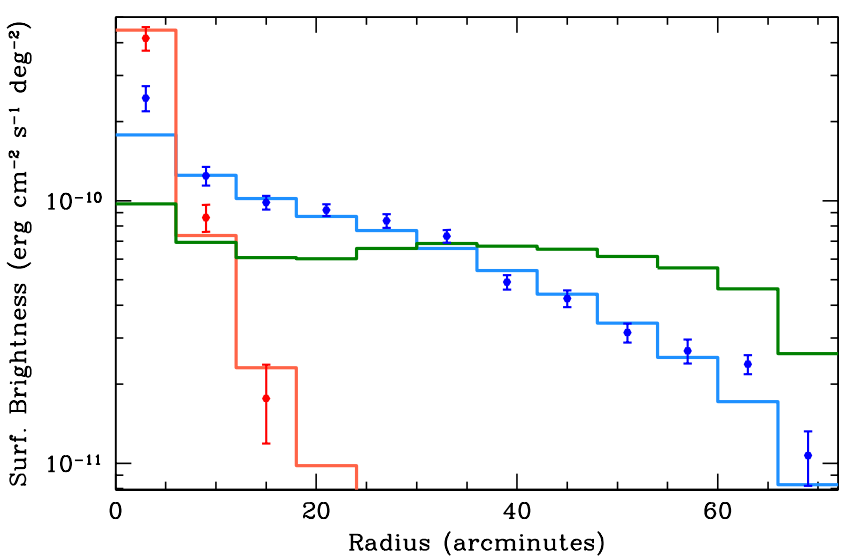

FIG. 15.- Nebula surface brightness profile indicating a remarkably uniform Fermi LAT profile. Red data points denote Suzaku X-ray data from Table 4 (statistical plus sytematic errors shown), while the red curve shows the Model 4 flux prediction in the $1-8 \mathrm{keV}$ band. Blue data points mark the H.E.S.S. flux from Table 3 , with the blue curve the $0.25-10 \mathrm{TeV}$ model prediction. The model 1-100 GeV band surface brightness is shown in green.

pulsar spin-down going into electrons is consistent with the data, though due to particle cooling at the current epoch magnetic field energy is $\approx 1 / 2 \times$ the particle energy in the nebula. This cooling also results in a higher magnetic than particle pressure, with a nearly isobaric particle pressure profile in the outer ten zones and the total pressure falling as $P_{\text {pwn }} \propto t^{-3}$. For the best fit model (Model 4) an initial spin period of $\approx 13 \mathrm{~ms}$ provides adequate pulsar spin-down energy to power the nebula, and combined with the braking index of $n=1.9$ this predicts an age of $40 \mathrm{kyr}$ and an expansion velocity of $2000 \mathrm{~km} \mathrm{~s}^{-1}$. The interior velocity profile is found to scale as $v(r) \sim r^{-0.5}$, while the magnetic field profile scales as $B(r, t) \propto r^{-0.7} \dot{E}(t)^{1 / 2}$, with the field falling from $\approx 400 \mu \mathrm{G}$ at the termination shock to $\approx 2 \mu \mathrm{G}$ in the far reaches of the nebula and the mean field scaling as $\bar{B} \propto t^{-1.6}$.

The Fermi LAT photon index of 1.4 connotes an electron index near the canonical 2.0. However, fitting to the X-ray/TeV and the higher energy portion of the electron spectrum, we find a somewhat softer power law index of $p \approx 2.2$. A relativistic Maxwellian electron spectrum provides a somewhat worse fit to the X-ray and H.E.S.S. data compared to a simple power-law injection, and the power-law case requires fewer fit parameters and better matches the Fermi LAT data. Regardless of injection spectrum, the existence of 10 $\mathrm{TeV}$ photons in the outer reaches of the nebula requires substantial particle diffusion; adopting a diffusion timescale of $\tau_{\text {esc }} \approx 90(R / 10 \mathrm{pc})^{2}\left(\mathrm{E}_{\mathrm{e}} / 100 \mathrm{TeV}\right)^{-1}$ year allows an adequate fit to the data. This is in contrast to the common assumption of a purely toroidal PWN magnetic field which could contain particles extremely efficiently. The model predicts a rather uniform Fermi LAT surface brightness out to $\sim 1^{\circ}$ from the pulsar, in good agreement with the recently discovered LAT source centered $0.5^{\circ}$ southwest of PSR J1826-1334, with extension $0.6 \pm 0.1^{\circ}$.

The multi-zone time-dependent SED model fitting approach applied in the paper extends simple one-zone models to allow investigation of spatially dependent properties such as flow speed, magnetic field, photon index, and flux levels. The growing number of sources with spatially resolved X-ray and VHE measurements (e.g. Vela-X, HESS J1303-631) are therefore prime targets for such multi-zone modeling.

Acknowledgments: We thank Stefan Funk and Marianne Lemoine-Goumard for helpful discussions on PWN modeling and Fermi LAT data. We also would like to thank the referee for very thorough and helpful comments. This work was supported in part by the U.S. Department of Energy contract to SLAC no. DE-AC02-76SF00515 and by NASA grant NNX10AP65G.

\section{REFERENCES}

Abdo, A. A. et al., 2010c, ApJ, 713, 146

Ackermann, M., et al. 2011, ApJ, 726, 35

Aharonian, F. A., Atoyan, A. M., \& Kifune, T. 1997, MNRAS, 291, 162

Aharonian, F., et al. 2006, A\&A, 460, 365

Blondin, J. M., Chevalier, R. A., \& Frierson, D. M. 2001, ApJ, 563, 806

Blumenthal, G. R., \& Gould, R. J. 1970, Reviews of Modern Physics, 42, 237

Bogovalov, S. V., \& Khangoulyan, D. V. 2002, Astronomy Letters, 28, 373

Bucciantini, N., Amato, E., Bandiera, R., Blondin, J. M., \& Del Zanna, L. 2004, A\&A, 423, 253

Bucciantini, N., Arons, J., \& Amato, E. 2010, arXiv:1005.1831

Clifton, T. R., Lyne, A. G., Jones, A. W., McKenna, J., \& Ashworth, M. 1992, MNRAS, 254, 177

Cordes, J. M., \& Lazio, T. J. W. 2002, arXiv:astro-ph/0207156

de Jager, O. C., \& et al. 2005, International Cosmic Ray Conference, 4, 239

de Jager, O. C., \& Djannati-Ataï, A. 2008, arXiv:0803.0116

Fang, J., \& Zhang, L. 2010, A\&A, 515, A20

Gelfand, J. D., Slane, P. O., \& Zhang, W. 2009, ApJ, 703, 2051

Gaensler, B. M., Schulz, N. S., Kaspi, V. M., Pivovaroff, M. J., \& Becker,

W. E. 2003, ApJ, 588, 441

Grondin, M.-H., et al. 2011, ApJ, 738, 42

Ishisaki, Y., et al. 2007, PASJ, 59, 113

Kennel, C. F., \& Coroniti, F. V. 1984, ApJ, 283, 694

Kennel, C. F., \& Coroniti, F. V. 1984, ApJ, 283, 710

Lemiere, A., Terrier, R., \& Djannati-Ataï, A. 2006, arXiv:astro-ph/0602436

Lemiere, A., Slane, P., Gaensler, B. M., \& Murray, S. 2009, ApJ, 706, 1269

Livingstone, M. A., Kaspi, V. M., Gavriil, F. P., Manchester, R. N., Gotthelf,

E. V. G., \& Kuiper, L. 2007, Ap\&SS, 308, 317
Longair, M. S. 2010, High Energy Astrophysics by Malcolm

S. Longair. Cambridge University Press, 2010. ISBN: 9780521756181 ,

Manchester RN, Taylor JH. 1977. Pulsars. San Francisco: Freeman

Ng, C.-Y., \& Romani, R. W. 2004, ApJ, 601, 479

Osborne, J. L., Pandey-Pommier, M., \& Udaya Shankar, N. 2009,

Astronomical Society of the Pacific Conference Series, 407, 349

Pacini, F., \& Salvati, M. 1973, ApJ, 186, 249

Pavlov, G. G., Kargaltsev, O., \& Brisken, W. F. 2008, ApJ, 675, 683

Porter, T. A., \& Strong, A. W. 2005, International Cosmic Ray Conference, 4,77

Press, W. H., Teukolsky, S. A., Vetterling, W. T., \& Flannery, B. P., 1992,

Cambridge: University Press, 2nd ed.

Reynolds, S. P., \& Chevalier, R. A. 1984, ApJ, 278, 630

Slane, P., Chen, Y., Schulz, N. S., Seward, F. D., Hughes, J. P., \& Gaensler, B. M. 2000, ApJ, 533, L29

Slane, P., Helfand, D. J., van der Swaluw, E., \& Murray, S. S. 2004, ApJ, 616, 403

Slane, P., Castro, D., Funk, S., Uchiyama, Y., Lemiere, A., Gelfand, J. D., Spitkovsky, A. 2008, ApJ, 682, L5

Tanaka, S. J., \& Takahara, F. 2010, ApJ, 715, 1248

Truelove, J. K., \& McKee, C. F. 1999, ApJS, 120, 299

Uchiyama, H., Matsumoto, H., Tsuru, T. G., Koyama, K., \& Bamba, A. 2009, PASJ, 61, 189

van der Swaluw, E., Achterberg, A., Gallant, Y. A., \& Tóth, G. 2001, A\&A, 380, 309

van der Swaluw, E., Downes, T. P., \& Keegan, R. 2004, A\&A, 420, 937 